\title{
Texture Mixer: A Network for Controllable Synthesis and Interpolation of Texture
}

\author{
Ning Yu ${ }^{1,2,4}$ Connelly Barnes ${ }^{3,4}$ Eli Shechtman ${ }^{3} \quad$ Sohrab Amirghodsi $^{3} \quad$ Michal Lukáč $^{3}$ \\ ${ }^{1}$ University of Maryland $\quad{ }^{2}$ Max Planck Institute for Informatics \\ ${ }^{3}$ Adobe Research $\quad{ }^{4}$ University of Virginia \\ ningyu@mpi-inf.mpg.de \\ connellyecs.virginia.edu \\ \{elishe, tamirgho, lukac\}eadobe.com
}

\begin{abstract}
This paper addresses the problem of interpolating visual textures. We formulate this problem by requiring (1) byexample controllability and (2) realistic and smooth interpolation among an arbitrary number of texture samples. To solve it we propose a neural network trained simultaneously on a reconstruction task and a generation task, which can project texture examples onto a latent space where they can be linearly interpolated and projected back onto the image domain, thus ensuring both intuitive control and realistic results. We show our method outperforms a number of baselines according to a comprehensive suite of metrics as well as a user study. We further show several applications based on our technique, which include texture brush, texture dissolve, and animal hybridization ${ }^{1}$.
\end{abstract}

\section{Introduction}

Many materials exhibit variation in local appearance, as well as complex transitions between different materials. Editing materials in an image, however, can be highly challenging due to the rich, spatially-varying material combinations as we see in the natural world. One general research challenge then is to attempt to enable these kinds of edits. In particular, in this paper, we focus on textures. We define "texture" as being an image-space representation of a statistically homogeneous material, captured from a top-down view. We further focus on allowing a user to both be able to accurately control the placement of textures, as well as create plausible transitions between them.

Because of the complex appearance of textures, creating transitions by interpolating between them on the pixel domain is difficult. Doing so naïvely results in unpleasant artifacts such as ghosting, visible seams, and obvious repetitions. Researchers in texture synthesis have therefore

\footnotetext{
${ }^{1}$ Demos, videos, code, data, models, and supplemental material are available at GitHub.
}

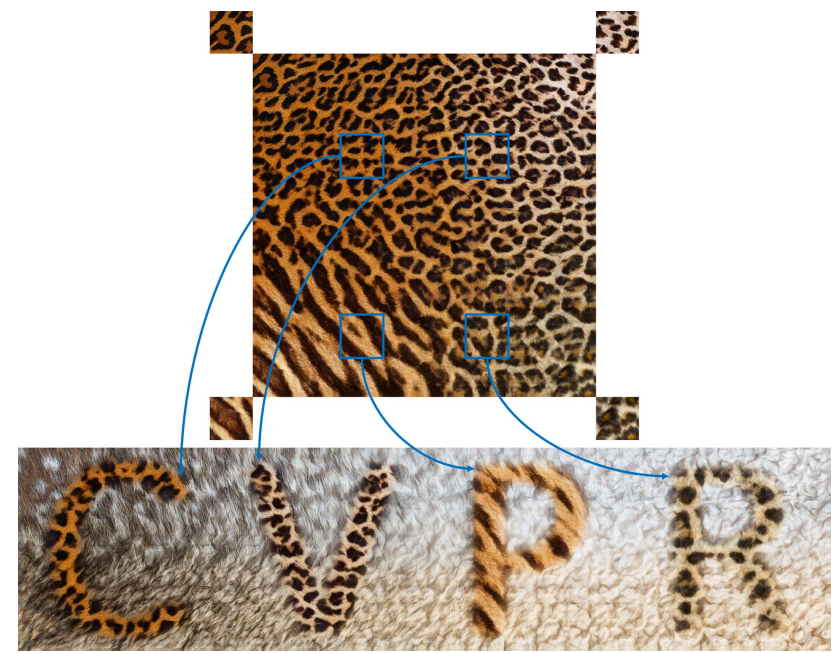

Figure 1. Texture interpolation and texture painting using our network on the animal texture dataset. The top part shows a $1024 \times 1024$ palette created by interpolating four source textures at the corners outside the palette. The bottom part shows a $512 \times 2048$ painting of letters with different textures sampled from the palette. The letters are interpolated by our method with the background, also generated by our interpolation.

developed sophisticated algorithms to address this problem. These may be divided to two families: non-parametric methods such as patch-based synthesis (e.g. [11, 10, 2]) and parametric methods (e.g. [17, 35]), including neural network synthesis approaches (e.g. [12, 40, 22, 29, 30]). Previously, researchers used sophisticated patch-based interpolation methods $[8,9]$ with carefully crafted objective functions. However, such approaches are extremely slow. Moreover, due to the hand-crafted nature of their objectives, they cannot learn from a large variety of textures in the natural world, and as we show in our comparisons are often brittle and frequently result in less pleasing transitions. Further, we are not aware of any existing feedforward neural network approaches that offer both fine-grained controllable synthesis and interpolation between multiple textures. User-controllable texture interpolation is substan- 
tially more challenging than ordinary texture synthesis, because it needs to incorporate adherence to user-provided boundary conditions and a smooth transition for the interpolated texture.

In our paper, we develop a neural network approach that we call "Texture Mixer," which allows for both user control and interpolation of texture. We define the interpolation of texture as a broad term, encompassing any combination of: (1) Either gradual or rapid spatial transitions between two or more different textures, as shown in the palette, the letters, and the background in Figure 1, and (2) Texture dissolve, where we can imagine putting two textures in different layers, and cross-dissolving them according to a usercontrolled transparency, as we show in our video. Previous neural methods can create interpolations similar to our dissolves by changing the latent variable [19, 23, 30, 31, 6]. Thus, in this paper we focus primarily on high-quality spatial interpolation: this requires textures to coexist in the same image plane without visible seams or spatial repetitions, which is more difficult to achieve. Our feedforward network is trained on a large dataset of textures and runs at interactive rates.

Our approach addresses the difficulty of interpolating between textures on the image domain by projecting these textures onto a latent domain where they may be linearly interpolated, and then decoding them back into the image domain to obtain the desired result. In order to satisfy the two goals of controllability and visual realism, we train our network simultaneously for both tasks. A reconstruction task ensures that when a texture is passed through an encoder and then a decoder (an autoencoder), the result will be similar to the input. This allows the user to specify texture at any given point of the output by example. An interpolation task uses a discriminator to ensure that linear interpolations of latent tensors also decode into plausible textures, so that the regions of the output not directly specified by the user are realistic and artifact-free. For this task, we can view our network as a conditional Generative Adversarial Network (GAN). In effect, we thus train an autoencoder and a conditional GAN at the same time, using shared weights and a shared latent space.

To perform the interpolation task, we take texture samples that user specifies, and project them into latent space using a learned encoder. Given these latent tensors, our network then uses three intuitive latent-space operations: tiling, interpolation, and shuffling. The tiling operation extends a texture spatially to any arbitrary size. The interpolation operation uses weighted combinations of two or more textures in latent domain. The shuffling operation swaps adjacent small squares within the latent tensor to reduce repetitions. These new latent tensors are then decoded to obtain the interpolated result.

Our main contributions are: (1) a novel interactive tech- nique that allows both user control and interpolation of texture; (2) several practical and creative applications based on our technique; (3) a new suite of metrics that evaluate user controllability, interpolation smoothness, and interpolation realism; and (4) the state-of-the-art performance superior to previous work both based on these metrics, and based on a user study if we consider them holistically.

\section{Related Work}

The problem of user-controllable texture interpolation has so far been under-explored. It is however closely related to several other problems, most significantly texture synthesis, inpainting, and stylization.

Texture synthesis algorithms can be divided into two families. The first one is parametric, with a generative texture model. These algorithms include older, non-neural methods [17, 35], and also more recent deep learning-based methods that are based on optimization [12, 13, 36, 38] or trained feedforward models [40, 22, 29, 30]. Where the underlying model allows spatially varying weights for combination, it may be used to cross-dissolve textures. However, we are not aware of any existing texture synthesis techniques in this family that enables spatial transition between different textures.

The second family of texture synthesis algorithms is nonparametric, in which the algorithm produces output that is optimized to be as close as possible to the input under some appearance measure [11, 41, 10, 27, 26, 33, 28, 42, 2, 8, 24$].$ These can be formulated to accept two different inputs and spatially vary which is being compared to, facilitating interpolation [8, 9]. As we mentioned before, such approaches are slow, and due to the hand-crafted nature of their objectives, they tend to be brittle.

Recently, generative adversarial networks (GANs) [14, $37,1,15]$ have shown improved realism in image synthesis and translation tasks [20, 48, 49]. GANs have also been used directly for texture synthesis [29, 21, 47], however, they were limited to a single texture they were trained on. A recent approach dubbed PSGAN [3] learns to synthesize a collection of textures present in a single photograph, making it more general and applicable to texture interpolation; it is not, however, designed for our problem as it cannot interpolate existing images. We show comparisons with PSGAN and it cannot reconstruct many input textures, even after running a sophisticated optimization or jointly associating PSGAN with an encoder. Moreover, PSGAN can suffer from mode collapse.

Texture synthesis and image inpainting algorithms are often closely related. A good hole filling algorithm needs to be able to produce some sort of transition between textures on opposite ends of the hole, and so may be used in a texture interpolation task. A few recent deep learning-based methods showed promising results [43, 45, 32, 44]. 


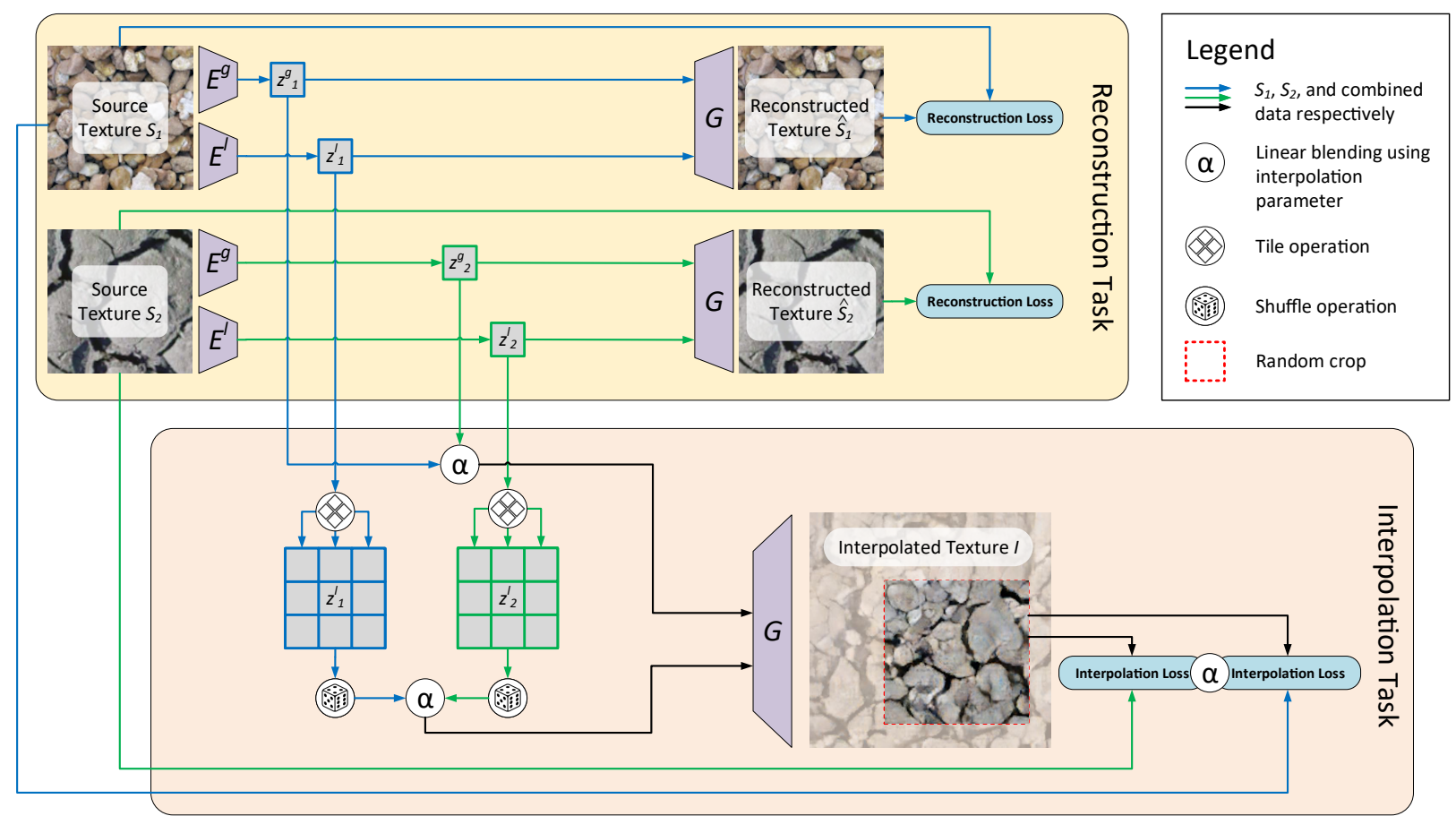

Figure 2. A diagram of our method. Background color highlights each of the tasks. Trapezoids represent trainable components that share weights if names match. Rounded rectangles represent the losses. Arrows and circles represent operations on tensor data.

Finally, some neural stylization approaches [13, 29, 19, 31] based on separating images into content and style components have shown that, by stylizing a noise content image, they can effectively synthesize texture [12]. By spatially varying the style component, texture interpolation may thus be achieved.

\section{Our network: Texture Mixer}

In this section, we explain how our network works. We first explain in Section 3.1 how our method is trained. We then show how our training losses are set up in Section 3.2. Finally, we explain in Section 3.3 how our method can be either tested or used by an end user.

\subsection{Training setup}

We aim to train our network simultaneously for two tasks: reconstruction and interpolation. The reconstruction task ensures that every input texture after being encoded and then decoded results in a similar texture. Meanwhile, the interpolation task ensures that interpolations of latent tensors are also decoded into plausible textures.

Our method can be viewed as a way of training a network containing both encoders and a generator, such that the generator is effectively a portion of a GAN. The network accepts a source texture $S$ as input. A global encoder $E^{g}(S)$ encodes $S$ into a latent vector $z^{g}$, which can also be viewed as a latent tensor with spatial size $1 \times 1$. A local encoder $E^{l}(S)$ encodes the source texture into a latent ten- sor $z^{l}$, which has a spatial size that is a factor $m$ smaller than the size of the input texture: we use $m=4$. The generator $G\left(z^{l}, z^{g}\right)$ concatenates $z^{l}$ and $z^{g}$, and can decode these latent tensors back into a texture patch, so that ideally $G\left(E^{l}(S), E^{g}(S)\right)=S$, which encompasses the reconstruction task. Our generator is fully convolutional, so that it can generate output textures of arbitrary size: the output texture size is directly proportional to the size of the local tensor $z^{l}$. A discriminator $D^{\text {rec }}$ is part of the reconstruction loss. An identical but separately trained discriminator $D^{\text {itp }}$ evaluates the realism of interpolation.

Note that in practice, our generator network is implemented as taking a global tensor as input, which has the same spatial size as the local tensor. This is because, for some applications of texture interpolation, $z^{g}$ can actually vary spatially. Thus, when we refer to $G$ taking a global latent vector $z^{g}$ with spatial size $1 \times 1$ as input, what we mean is that this $z^{g}$ vector is first repeated spatially to match the size of $z^{l}$, and the generator is run on the result.

We show the full training setup in Figure 2. We will also explain our setup in terms of formulas here. As is shown in the upper-left of Figure 2, the network is given two real source texture images $S_{1}$ and $S_{2}$ from the real texture dataset $\mathcal{S}$. Each local encoder $E^{l}$ encodes $S_{i}(i \in\{1,2\})$ to a local latent tensor $z_{i}^{l}=E^{l}\left(S_{i}\right)$. Meanwhile, each global encoder $E^{g}$ encodes $S_{i}$ to a global latent vector $z_{i}^{g}$, denoted as $z_{i}^{g}=E^{g}\left(S_{i}\right)$. These latent variables are shown in green and blue boxes in the upper-left of Figure 2. 
For the reconstruction task, we then evaluate the reconstructed texture image $\hat{S}_{i}=G\left(z_{i}^{l}, z_{i}^{g}\right)$. These are shown in the upper center of Figure 2. For each reconstructed image $\hat{S}_{i}$, we then impose a weighted sum of three losses against the original texture $S_{i}$. We describe these losses in more detail later in Section 3.2.

For the interpolation task, we pose the process of multiple texture interpolation as a problem of simultaneously (1) synthesizing a larger texture, and (2) interpolating between two different textures. In this manner, the network learns to perform well for both single and multiple texture synthesis. For single texture synthesis, we enlarge the generated images by a factor of $3 \times 3$. We do this by tiling $z_{i}^{l}$ spatially by a factor of $3 \times 3$. We denote this tiling by $T\left(z_{i}^{l}\right)$, and indicate tiling by a tile icon in the lower-left of Figure 2. We chose the factor 3 because this is the smallest integer that can synthesize transitions over the four edges of $z_{i}^{l}$. Such a small tiling factor minimizes computational cost. The tiling operation can be beneficial for regular textures. However, in semiregular or stochastic textures, the tiling introduces two artifacts: undesired spatial repetitions, and undesired seams on borders between tiles.

We reduce these artifacts by applying a random shuffling to the tiled latent tensors $T\left(z_{i}^{l}\right)$. In Figure 2, this shuffling operation is indicated by a dice icon. Random shuffling in the latent space not only results in more varied decoded image appearance and thus reduces visual repetition, but also softens seams by spatially swapping pixels in the latent space across the border of two $z_{i}^{l}$ tensors.

We implement the random shuffling by row and column swapping over several scales from coarse to fine. For this coarse to fine process, we use scales that are powers of two: $s_{i}=2^{i}$ for $i=0,2, \ldots, n$. We set the coarsest scale $n$ to give a scale $s_{n}$ that is half the size of the local tensor $z_{i}^{l}$. For each scale $s_{i}$, we define a grid over the tiled latent tensor $T\left(z^{l}\right)$, where each grid cell has size $s_{i} \times s_{i}$. For each scale $s_{i}$, we then apply a random shuffling on cells of the grid for that scale: we denote this by $P_{i}$. This shuffling proceeds through grid rows first in top-down and then bottom-up order: each row is randomly swapped with the succeeding row with probability 0.5 . Similarly, this is repeated on grid columns, with column swapping from left to right and right to left. Thus, the entire shuffling operation is:

$$
P\left(T\left(z_{i}^{l}\right)\right)=P_{0} \circ P_{1} \circ \cdots \circ P_{n}\left(T\left(z_{i}^{l}\right)\right)
$$

We visualize this shuffling procedure in the supplementary material. We also want the synthesized texture to be able to transit smoothly between regions where there are userspecified texture constraints and regions where there are none. Thus, we override the original $z_{i}^{l}$ without shuffling at the 4 corners of the tiled latent tensor. We denote such shuffling with corner overriding as $\tilde{P}\left(T\left(z_{i}^{l}\right)\right)$.

If we apply the fully convolutional generator $G$ to a net- work trained using a single input texture and the above shuffling process, it will work for single texture synthesis. However, for multiple texture interpolation, we additionally apply interpolation in the latent space before calling $G$, as inspired by $[30,19,3]$. We randomly sample an interpolation parameter $\alpha \sim U[0,1]$, and then interpolate the latent tensors using $\alpha$. This is shown by the circles labeled with $\alpha$ in Figure 2. We linearly blend the shuffled local tensors $\tilde{P}\left(T\left(z_{1}^{l}\right)\right)$ and $\left.\tilde{P}\left(T\left(z_{2}^{l}\right)\right)\right)$, which results in the final interpolated latent tensor $Z^{l}$ :

$$
Z^{l}=\alpha \tilde{P}\left(T\left(z_{1}^{l}\right)\right)+(1-\alpha) \tilde{P}\left(T\left(z_{2}^{l}\right)\right)
$$

In the same way, we blend $z_{1}^{g}$ and $z_{2}^{g}$ to obtain

$$
Z^{g}=\alpha z_{1}^{g}+(1-\alpha) z_{2}^{g}
$$

Finally, we feed the tiled and blended tensors into the generator $G$ to obtain an interpolated texture image $I=$ $G\left(Z^{l}, Z^{g}\right)$, which is shown on the right in Figure 2. From the interpolated texture, we take a random crop of the same size as the input textures. The crop is shown in the red dotted lines in Figure 2. The crop is then compared using appropriately $\alpha$-weighted losses to each of the source textures. We use spatially uniform weights $\alpha$ at training time because all the real-world examples are spatially homogeneous and we do not want our adversarial discriminator to detect our synthesized texture due to it having spatial variation. In contrast, at testing time, we use spatially varying weights.

\subsection{Training losses}

For the reconstruction task, we use three losses. The first loss is a pixel-wise $L_{1}$ loss against each input $S_{i}$. The second loss is a Gram matrix loss against each input $S_{i}$, based on an ImageNet-pretrained VGG-19 model. We define the Gram loss $L_{\text {Gram }}$ in the same manner as Johnson et al. [22], and use the features relu $i_{-} 1$ for $i=1, \ldots, 5$. The third loss is an adversarial loss $L_{\text {adv }}$ based on WGAN-GP [15], where the reconstruction discriminator $D^{\text {rec }}$ tries to classify whether the reconstructed image is from the real source texture set or generated by the network. The losses are:

$$
\begin{gathered}
L_{\mathrm{pix}}^{\mathrm{rec}}=\left\|\hat{S}_{1}-S_{1}\right\|_{1}+\left\|\hat{S}_{2}-S_{2}\right\|_{1} \\
L_{\mathrm{Gram}}^{\mathrm{rec}}=L_{\mathrm{Gram}}\left(\hat{S}_{1}, S_{1}\right)+L_{\mathrm{Gram}}\left(\hat{S}_{2}, S_{2}\right) \\
L_{\mathrm{adv}}^{\mathrm{rec}}=L_{\mathrm{adv}}\left(\hat{S}_{1}, S_{1} \mid D^{\mathrm{rec}}\right)+L_{\mathrm{adv}}\left(\hat{S}_{2}, S_{2} \mid D^{\mathrm{rec}}\right)
\end{gathered}
$$

The $L_{\text {adv }}$ term is defined from WGAN-GP [15] as:

$$
L_{\text {adv }}(A, B \mid D)=D(A)-D(B)+G P(A, B \mid D)
$$

Here $A$ and $B$ are a pair of input images, $D$ is the adversarially trained discriminator, and $G P(\cdot)$ is the gradient penalty regularization term. 

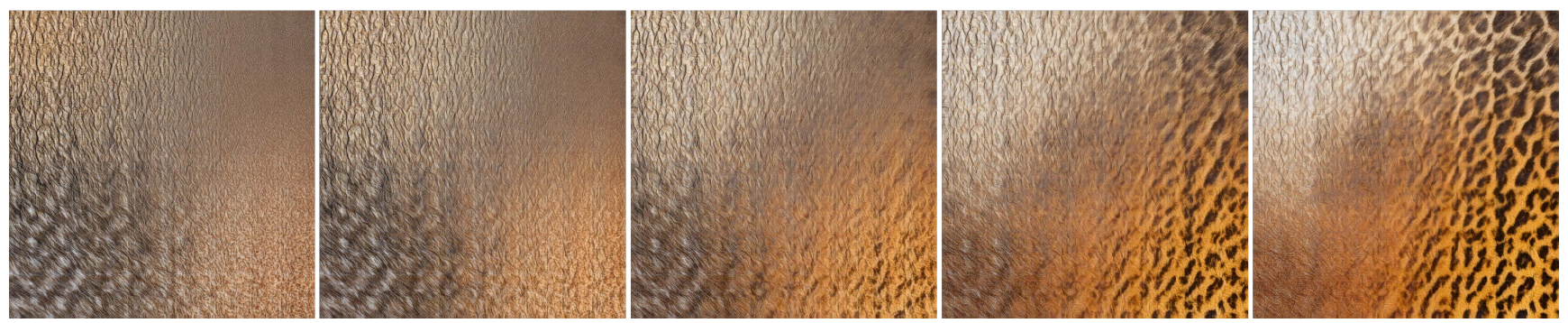

Figure 3. A sequence of dissolve video frame samples with size $1024 \times 1024$ on the animal texture dataset, where each frame is also with effect of interpolation.

For the interpolation task, we expect the large interpolated texture image to be similar to some combination of the two input textures. Specifically, if $\alpha=1$, the interpolated image should be similar to source texture $S_{1}$, and if $\alpha=0$, it should be similar to $S_{2}$. However, we do not require pixelwise similarity, because that would encourage ghosting. We thus impose only a Gram matrix and an adversarial loss. We select a random crop $I_{\text {crop }}$ from the interpolated texture image. Then the Gram matrix loss for interpolation is defined as an $\alpha$-weighted loss to each source texture:

$$
L_{\text {Gram }}^{\text {itp }}=\alpha L_{\text {Gram }}\left(I_{\text {crop }}, S_{1}\right)+(1-\alpha) L_{\text {Gram }}\left(I_{\text {crop }}, S_{2}\right)
$$

Similarly, we adversarially train the interpolation discriminator $D^{\text {itp }}$ for the interpolation task to classify whether its input image is from the real source texture set or whether it is a synthetically generated interpolation:

$$
L_{\text {adv }}^{\text {itp }}=\alpha L_{\text {adv }}\left(I_{\text {crop }}, S_{1} \mid D^{\mathrm{itp}}\right)+(1-\alpha) L_{\text {adv }}\left(I_{\text {crop }}, S_{2} \mid D^{\text {itp }}\right)
$$

Our final training objective is

$$
\begin{array}{r}
\min _{E^{l}, E^{g}, G} \max _{D^{\text {rec }}, D^{\text {itp }}} \underset{S_{1}, S_{2} \sim \mathcal{S}}{\mathbb{E}}\left(\lambda_{1} L_{\text {pix }}^{\text {rec }}+\lambda_{2} L_{\text {Gram }}^{\text {rec }}+\lambda_{3} L_{\text {adv }}^{\text {rec }}\right. \\
\left.+\lambda_{4} L_{\text {Gram }}^{\text {itp }}+\lambda_{5} L_{\text {adv }}^{\text {itp }}\right)
\end{array}
$$

where $\lambda_{1}=100, \lambda_{2}=\lambda_{4}=0.001$, and $\lambda_{3}=\lambda_{5}=1$ are used to balance the order of magnitude of each loss term, which are not sensitive to dataset.

We provide details related to our training and architecture in the supplementary document, such as how we used progressive growing during training [23].

\subsection{Testing and user interactions}

At testing time, we can use our network in several different ways: we can interpolate sparsely placed textures, brush with textures, dissolve between textures, and hybridize different animal regions in one image. Each of these applications utilizes spatially varying interpolation weights.

Interpolation of sparsely placed textures. This option is shown in the palette and background in Figure 1. In this scenario, one or more textures are placed down by the user in the image domain. These textures are each encoded to latent domain.

In most cases, given input textures, our method is able to achieve inherent boundary matching and continuity. However, because of the trade-off between reconstruction and interpolation losses, there might be a slight mismatch in some cases. To make the textures better agree at boundary conditions, we postprocess our images as follows. Suppose that the user places a source textured region as a boundary condition. We first replace the reconstructed regions with the source texture. Then, within the source texture, we use graph cuts [27] to determine an optimal seam where we can cut between the source texture and the reconstruction. Finally, we use Poisson blending [34] to minimize the visibility of this seam.

Texture brush. We can allow the user to brush with texture as follows. We assume that there is a textured background region, which we have encoded to latent space. The user can select any texture to brush with, by first encoding the brush texture and then brushing in the latent space. For example, in Figure 1 we show an example of selecting a texture from a palette created by interpolating four sparsely created textures. We find the brush texture's latent domain tensors, and apply them using a Gaussian-weighted brush. Here full weight in the brush causes the background latent tensors to be replaced entirely, and other weights cause a proportionately decreased effect. The brush can easily be placed spatially because the latent and image domains are aligned with a resizing factor $m$ related to the architecture. We show more results in the supplementary material.

Texture dissolve. We can create a cross-dissolve effect between any two textures by encoding them both to latent domain and then blending between them using blending weights that are spatially uniform. This effect is best visualized in a video, where time controls the dissolve effect. Please see our supplementary video for such results. Figure 3 shows a sequence of video frame samples with gradually varying weights.

Animal hybridization. We generalize texture interpolation into a more practical and creative application - animal hybridization. Figure 4 shows an example. Given two aligned animal regions in one image and a hole over the 


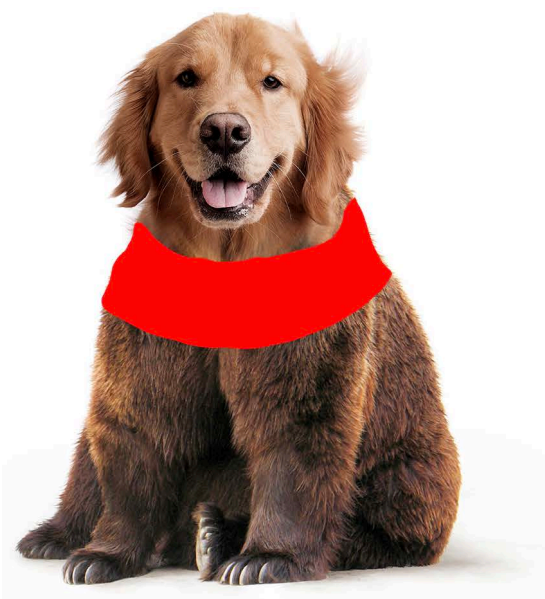

Input: aligned animal region with hole

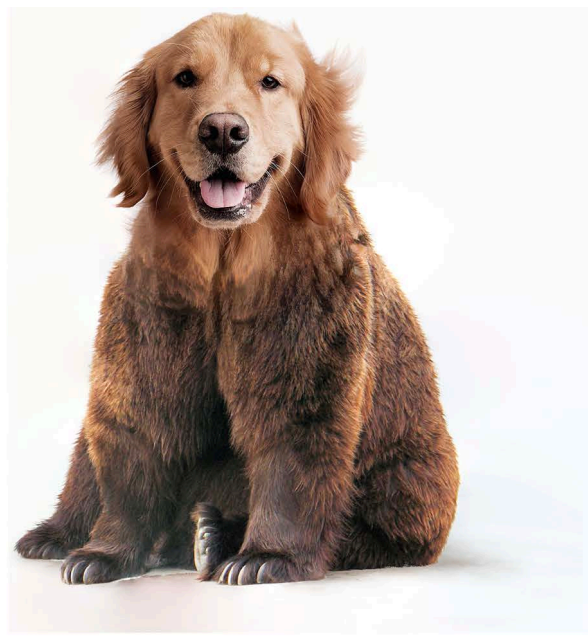

Our result

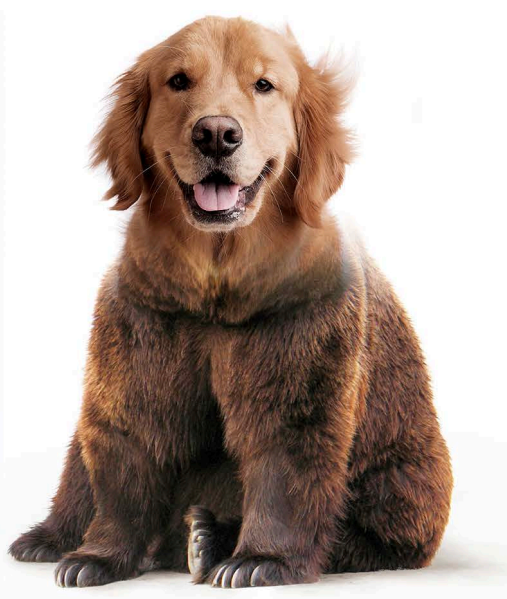

Naïve $\boldsymbol{\alpha}$-blending result

Figure 4. An animal hybridization example of size $1260 \times 1260$ between a dog and a bear. Our interpolation between the two animal furs is smoother, has less ghosting, and is more realistic than that of the Naïve $\alpha$-blending.

transition region, we can sample source texture patches adjacent to the hole and conduct spatial interpolation among those textures. We fill the hole using our interpolated texture. Finally, we use graph cuts [27] and Poisson blending [34] to postprocess the boundaries. Technical details and more examples are shown in the supplemental material.

\section{Experiments}

In this section, we demonstrate experimental comparisons. We first introduce our own datasets in Section 4.1. We then present in Section 4.2 a suite of evaluation metrics for interpolation quality. In Section 4.3 we list and compare against several leading methods from different categories on the task of texture interpolation. In Section 4.4 we describe a user study as a holistic comparison. Finally, we conduct in Section 4.5 the ablation study by comparing against three simplified versions of our own method.

We propose to learn a model per texture category rather than a universal model because: (1) there are no real-world examples that depict interpolation between distinct texture categories; (2) there is no practical reason to interpolate across categories, e.g., fur and gravel; and (3) like with other GANs, a specific model per category performs better than a universal one due to the model's capacity limit.

\subsection{Datasets}

Training to interpolate frontal-parallel stationary textures of a particular category requires a dataset with a rich set of examples to represent the intra-variability of that category. Unfortunately, most existing texture datasets such as DTD [7] are intended for texture classification tasks, and do not have enough samples per category (only 120 in the case of DTD) to cover the texture appearance space with sufficient density.
Therefore, we collected two datasets of our own: (1) the earth texture dataset contains Creative Commons images from Flickr, which we randomly split into 896 training and 98 testing images; (2) the animal texture dataset contains images from Adobe Stock, randomly split into 866 training and 95 testing images. All textures are real-world RGB photos with arbitrary sizes larger than $512 \times 512$. Examples from both are shown in our figures throughout the paper.

We further augmented all our training and testing sets by applying: (1) color histogram matching with a random reference image in the same dataset; (2) random geometric transformations including horizontal and vertical mirroring, random in-plane rotation and downscaling (up to $\times 4$ ); and (3) randomly cropping a size of $128 \times 128$. In this way, we augmented 1,000 samples for each training image and 100 samples for each testing image.

\subsection{Evaluation}

We will compare previous work with ours, and also do an ablation study on our own method. In order to fairly compare all methods, we use a horizontal interpolation task. Specifically, we randomly sampled two $128 \times 128$ squares from the test set. We call these the side textures. We placed them as constraints on either end of a $128 \times 1024$ canvas. We then used each method to produce the interpolation on the canvas, configuring each method to interpolate linearly where such option is available.

To the best of our knowledge, there is no standard method to quantitatively evaluate texture interpolation. We found existing generation evaluation techniques [37, 18, 4, 23] inadequate for our task. We, therefore, developed a suite of metrics that evaluate three aspects we consider crucial for our task: (1) user controllability, (2) interpolation smoothness, and (3) interpolation realism. We now discuss these. 
User controllability. For interpolation to be considered controllable, it has to closely reproduce the user's chosen texture at the user's chosen locations. In our experiment, we measure this as the reconstruction quality for the side textures. We average the LPIPS perceptual similarity measure [46] for the two side textures. We call this Side Perceptual Distance (SPD).

We also would like the center of the interpolation to be similar to both side textures. To measure this, we consider the Gram matrix loss [22] between the central $128 \times 128$ crop of the interpolation and the side textures. We report the sum of distances from the center crop to the two side textures, normalized by the Gram distance between the two. We call this measure the Center Gram Distance (CGD).

Interpolation smoothness. Ideally, we would like the interpolation to follow the shortest path between the two side textures. To measure this, we construct two difference vectors of Gram matrix features between the left side texture and the center crop, and between the center crop and the right side texture, and measure the cosine distance between the two vectors. We expect this Centre Cosine distance $(C C D)$ to be minimized.

For smoothness, the appearance change should be gradual, without abrupt changes such as seams and cuts. To measure such, we train a seam classifier using real samples from the training set as negative examples, and where we create synthetic seams by concatenating two random textures as positive examples. We run this classifier on the center crop. We call this the Center Seam Score (CSS). The architecture and training details of seam classifier are the same as those of $D^{\text {rec }}$ and $D^{\text {itp }}$.

Interpolation realism. The texture should also look realistic, like the training set. To measure this, we chose the Inception Score [37] and Sliced Wasserstein Distance (SWD) [23], and apply them on the center crops. This gives Center Inception Score (CIS) and Center SWD, respectively. For CIS, we use the state-of-the-art InceptionResNet-v2 inception model architecture [39] finetuned with our two datasets separately.

We also found these metrics do not capture undesired repetitions, a common texture synthesis artifact. We, therefore, trained a repetition classifier for this purpose. We call this the Center Repetition Score (CRS). The architecture and training details of repetition classifier are almost the same as those of the seam classifier except the input image size is $128 \times 256$ instead of $128 \times 128$, where the negative examples are random crops of size $128 \times 256$ from real datasets and the positive examples are horizontally tiled twice from random crops of size $128 \times 128$ from real datasets.

\subsection{Comparisons}

We compare against several leading methods from different categories on the task of texture interpolation. These

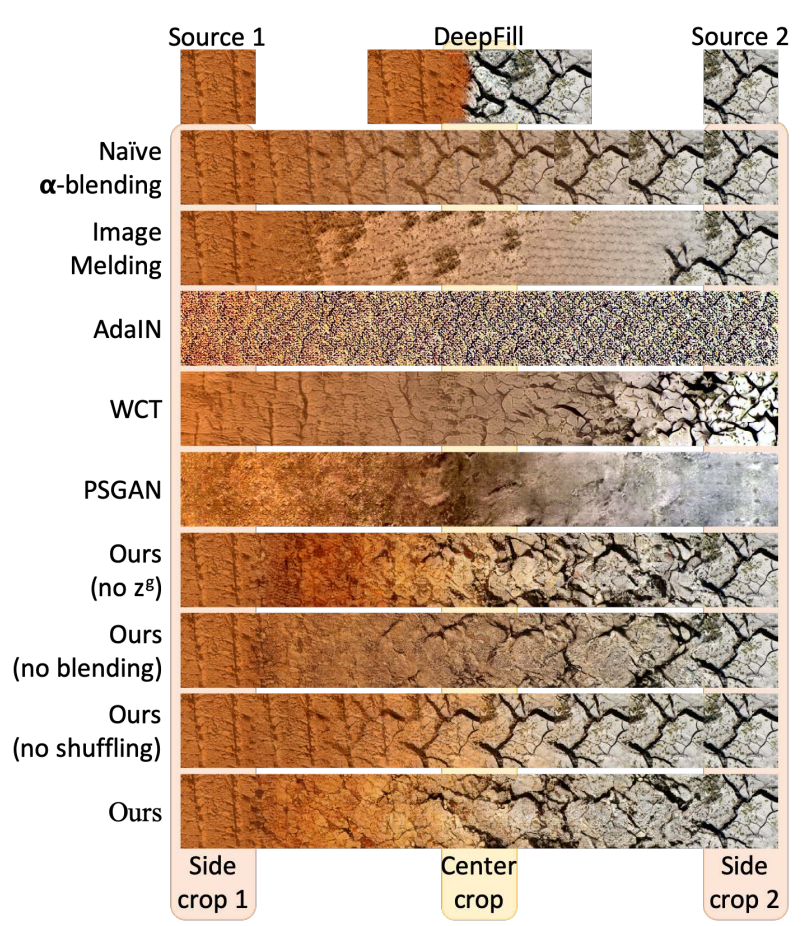

Figure 5. Qualitative demonstrations and comparisons of horizontal interpolation in the size of $128 \times 1024$ on the earth texture samples. We use the two side crops with the orange background for SPD measurement, and the center crop with the light yellow background for the other proposed quantitative evaluations. For the DeepFill [45] method, since the default design is not suitable for inpainting a wide hole due to lack of such ground truth, we instead test it on a shorter interpolation of size $128 \times 384$.

include: naïve $\alpha$-blending, Image Melding [8] as a representative of patch-based techniques, two neural stylization methods - AdaIN [19] and WCT [31], a recent deep holefilling method called DeepFill [45], and PSGAN [3] which is the closest to ours but without user control. Most these had to be adapted for our task. See more details in the supplementary material. Fig. 5 contains a qualitative comparison between the different methods. Note that in this example: (1) the overly sharp interpolation of DeepFill, (2) the undesired ghosting and repetition artifacts of naïve $\alpha$ blending and ours (no shuffling), (3) the incorrect reconstruction and less relevant interpolation of AdaIN, WCT, and PSGAN, (4) the appearance mismatch between source and interpolation of Image Melding, (5) the lack of smoothness of ours (no $z^{g}$ ), and (6) the undesired fading of ours (no blending). More qualitative comparisons are shown in the supplementary material. We also report qualitative results, including the user study and the ablation experiments, in Table 1, that contains average values for the two datasets - earth texture and animal texture. Figure 6 summarizes the quantitative comparisons. 
Table 1. Quantitative evaluation averaging over the earth texture and animal texture datasets. We highlighited the best, second best and very high values for each metric. We also indicate for each whether higher $(\Uparrow)$ or lower $(\Downarrow)$ values are more desirable.

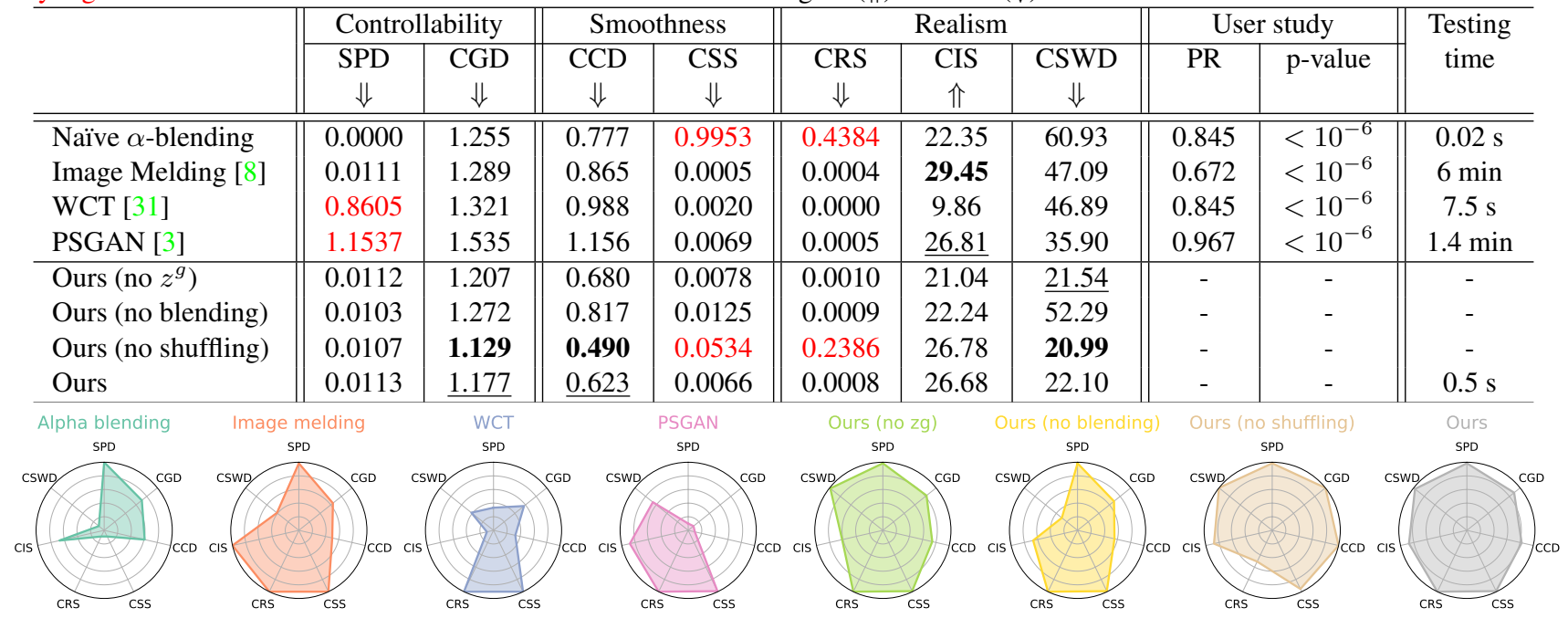

Figure 6. Radar charts visualizing Table 1. Values have been normalized to the unit range, and axes inverted so that higher value is always better. The first four are baseline methods and next three ablation candidates, with the last entry representing our full method. Our method scores near-top marks all around and shows balanced performance according to all metrics.

\subsection{User study}

We also conducted a user study on Amazon Mechanical Turk. We presented the users with a binary choice, asking them if they aesthetically prefer our method or one of the baseline methods on a random example from the horizontal interpolation task. The user study webpage and sanity check (to guarantee the effectiveness of users' feedback) are shown in the supplementary material. For each method pair, we sampled 90 examples and collected 5 independent user responses per example. Tallying the user votes, we get 90 results per method pair. We assumed a null hypothesis that on average, our method will be preferred by 2.5 users for a given method pair. We used a one-sample permutation t-test to measure $\mathrm{p}$-values, using $10^{6}$ permutations, and found the $\mathrm{p}$-values for the null hypothesis are all $<10^{-6}$. This indicates that the users do prefer one method over another. To quantify this preference, we count for each method pair all the examples where at least 3 users agree in their preference, and report a preference rate $(P R)$ which shows how many of the preferences were in our method's favor. Both PR and the p-values are listed in Table 1.

\subsection{Ablation study}

We also compare against simplified versions of our method. The qualitative results for this comparison are shown in Figure 5. We report quantitative result numbers in Table 1, and visualized them in Figure 6. We ablate the following components:

Remove $\mathbf{z}^{\mathbf{g}}$. The only difference between $z^{g}$ and $z^{l}$ is in the tiling and shuffling for $z^{l}$. However, if we remove $z^{g}$, we find texture transitions are less smooth and gradual.
Remove texture blending during training. We modify our method so that the interpolation task during training is performed only upon two identical textures. This makes the interpolation discriminator $D^{\text {itp }}$ not be aware of the realism of blended samples, so testing realism deteriorates.

Remove random shuffling. We skip the shuffling operation in latent space and only perform blending during training. This slightly improves realism and interpolation directness, but causes visually disturbing repetitions.

\section{Conclusion}

We presented a novel method for controllable interpolation of textures. We were able to satisfy the criteria of controllability, smoothness, and realism. Our method outperforms several baselines on our newly collected datasets. As we see in Figure 6, although some baseline method may achieve better results than ours on one of the evaluation criteria, they usually fail on the others. In contrast, our method has consistent high marks in all evaluation categories. The user study also shows the users overwhelmingly prefer our method to any of the baselines. We have also demonstrated several applications based on this technique and hope it may become a building block of more complex workflows.

\section{Acknowledgement}

The authors acknowledge the Maryland Advanced Research Computing Center for providing computing resources and acknowledge the photographers for licensing photos under Creative Commons or public domain. 


\section{References}

[1] Martin Arjovsky, Soumith Chintala, and Léon Bottou. Wasserstein gan. arXiv preprint arXiv:1701.07875, 2017. 2

[2] Connelly Barnes, Eli Shechtman, Adam Finkelstein, and Dan B Goldman. Patchmatch: A randomized correspondence algorithm for structural image editing. ACM Transactions on Graphics (ToG), 28(3):24, 2009. 1, 2

[3] Urs Bergmann, Nikolay Jetchev, and Roland Vollgraf. Learning texture manifolds with the periodic spatial GAN. In Proceedings of the 34th International Conference on Machine Learning, pages 469-477, 2017. 2, 4, 7, 8, 13

[4] Mikołaj Bińkowski, Dougal J Sutherland, Michael Arbel, and Arthur Gretton. Demystifying mmd gans. arXiv preprint arXiv:1801.01401, 2018. 6

[5] Richard H Byrd, Peihuang Lu, Jorge Nocedal, and Ciyou Zhu. A limited memory algorithm for bound constrained optimization. SIAM Journal on Scientific Computing, 16(5):1190-1208, 1995. 13

[6] Dongdong Chen, Lu Yuan, Jing Liao, Nenghai Yu, and Gang Hua. Stylebank: An explicit representation for neural image style transfer. In Proceedings of the IEEE Conference on Computer Vision and Pattern Recognition, pages 18971906, 2017. 2

[7] M. Cimpoi, S. Maji, I. Kokkinos, S. Mohamed, , and A. Vedaldi. Describing textures in the wild. In Proceedings of the IEEE Conf. on Computer Vision and Pattern Recognition (CVPR), 2014. 6

[8] Soheil Darabi, Eli Shechtman, Connelly Barnes, Dan B Goldman, and Pradeep Sen. Image melding: Combining inconsistent images using patch-based synthesis. ACM Trans. Graph., 31(4):82-1, 2012. 1, 2, 7, 8, 13

[9] Olga Diamanti, Connelly Barnes, Sylvain Paris, Eli Shechtman, and Olga Sorkine-Hornung. Synthesis of complex image appearance from limited exemplars. ACM Transactions on Graphics (TOG), 34(2):22, 2015. 1, 2

[10] Alexei A Efros and William T Freeman. Image quilting for texture synthesis and transfer. In Proceedings of the 28th annual conference on Computer graphics and interactive techniques, pages 341-346. ACM, 2001. 1, 2

[11] Alexei A Efros and Thomas K Leung. Texture synthesis by non-parametric sampling. In iccv, page 1033. IEEE, 1999. 1,2

[12] Leon Gatys, Alexander S Ecker, and Matthias Bethge. Texture synthesis using convolutional neural networks. In $A d$ vances in Neural Information Processing Systems, pages 262-270, 2015. 1, 2, 3

[13] Leon A Gatys, Alexander S Ecker, and Matthias Bethge. Image style transfer using convolutional neural networks. In Proceedings of the IEEE Conference on Computer Vision and Pattern Recognition, pages 2414-2423, 2016. 2, 3

[14] Ian Goodfellow, Jean Pouget-Abadie, Mehdi Mirza, Bing $\mathrm{Xu}$, David Warde-Farley, Sherjil Ozair, Aaron Courville, and Yoshua Bengio. Generative adversarial nets. In Advances in neural information processing systems, pages 2672-2680, 2014. 2
[15] Ishaan Gulrajani, Faruk Ahmed, Martin Arjovsky, Vincent Dumoulin, and Aaron C Courville. Improved training of wasserstein gans. In Advances in Neural Information Processing Systems, pages 5767-5777, 2017. 2, 4

[16] Kaiming He, Xiangyu Zhang, Shaoqing Ren, and Jian Sun. Deep residual learning for image recognition. In Proceedings of the IEEE conference on computer vision and pattern recognition, pages 770-778, 2016. 12

[17] David J Heeger and James R Bergen. Pyramid-based texture analysis/synthesis. In Proceedings of the 22nd annual conference on Computer graphics and interactive techniques, pages 229-238. ACM, 1995. 1, 2

[18] Martin Heusel, Hubert Ramsauer, Thomas Unterthiner, Bernhard Nessler, and Sepp Hochreiter. Gans trained by a two time-scale update rule converge to a local nash equilibrium. In Advances in Neural Information Processing Systems, pages 6626-6637, 2017. 6

[19] Xun Huang and Serge J Belongie. Arbitrary style transfer in real-time with adaptive instance normalization. In $I C C V$, pages 1510-1519, 2017. 2, 3, 4, 7, 13

[20] Phillip Isola, Jun-Yan Zhu, Tinghui Zhou, and Alexei A Efros. Image-to-image translation with conditional adversarial networks. In IEEE Conference on Computer Vision and Pattern Recognition, 2017. 2

[21] Nikolay Jetchev, Urs Bergmann, and Roland Vollgraf. Texture synthesis with spatial generative adversarial networks. arXiv preprint arXiv:1611.08207, 2016. 2

[22] Justin Johnson, Alexandre Alahi, and Li Fei-Fei. Perceptual losses for real-time style transfer and super-resolution. In European Conference on Computer Vision, pages 694-711. Springer, 2016. 1, 2, 4, 7, 14

[23] Tero Karras, Timo Aila, Samuli Laine, and Jaakko Lehtinen. Progressive growing of gans for improved quality, stability, and variation. arXiv preprint arXiv:1710.10196, 2017. 2, 5, $6,7,12$

[24] Alexandre Kaspar, Boris Neubert, Dani Lischinski, Mark Pauly, and Johannes Kopf. Self tuning texture optimization. In Computer Graphics Forum, volume 34, pages 349-359. Wiley Online Library, 2015. 2

[25] Diederik P Kingma and Jimmy Ba. Adam: A method for stochastic optimization. arXiv preprint arXiv:1412.6980, 2014. 12

[26] Vivek Kwatra, Irfan Essa, Aaron Bobick, and Nipun Kwatra. Texture optimization for example-based synthesis. In ACM Transactions on Graphics (ToG), volume 24, pages 795-802. ACM, 2005. 2

[27] Vivek Kwatra, Arno Schödl, Irfan Essa, Greg Turk, and Aaron Bobick. Graphcut textures: image and video synthesis using graph cuts. ACM Transactions on Graphics (ToG), 22(3):277-286, 2003. 2, 5, 6, 14

[28] Sylvain Lefebvre and Hugues Hoppe. Appearance-space texture synthesis. In ACM Transactions on Graphics (TOG), volume 25, pages 541-548. ACM, 2006. 2

[29] Chuan Li and Michael Wand. Precomputed real-time texture synthesis with markovian generative adversarial networks. In European Conference on Computer Vision, pages 702-716. Springer, 2016. 1, 2, 3 
[30] Yijun Li, Chen Fang, Jimei Yang, Zhaowen Wang, Xin Lu, and Ming-Hsuan Yang. Diversified texture synthesis with feed-forward networks. In Proc. CVPR, 2017. 1, 2, 4

[31] Yijun Li, Chen Fang, Jimei Yang, Zhaowen Wang, Xin Lu, and Ming-Hsuan Yang. Universal style transfer via feature transforms. In Advances in Neural Information Processing Systems, pages 386-396, 2017. 2, 3, 7, 8, 13

[32] Guilin Liu, Fitsum A Reda, Kevin J Shih, Ting-Chun Wang, Andrew Tao, and Bryan Catanzaro. Image inpainting for irregular holes using partial convolutions. In Proceedings of the European Conference on Computer Vision (ECCV), 2018. 2, 13

[33] Wojciech Matusik, Matthias Zwicker, and Frédo Durand. Texture design using a simplicial complex of morphable textures. In ACM Transactions on Graphics (TOG), volume 24, pages 787-794. ACM, 2005. 2

[34] Patrick Pérez, Michel Gangnet, and Andrew Blake. Poisson image editing. ACM Transactions on graphics (TOG), 22(3):313-318, 2003. 5, 6, 14

[35] Javier Portilla and Eero P Simoncelli. A parametric texture model based on joint statistics of complex wavelet coefficients. International journal of computer vision, 40(1):4970, 2000. 1, 2

[36] Eric Risser, Pierre Wilmot, and Connelly Barnes. Stable and controllable neural texture synthesis and style transfer using histogram losses. arXiv preprint arXiv:1701.08893, 2017. 2

[37] Tim Salimans, Ian Goodfellow, Wojciech Zaremba, Vicki Cheung, Alec Radford, and Xi Chen. Improved techniques for training gans. In Advances in Neural Information Processing Systems, pages 2234-2242, 2016. 2, 6, 7

[38] Omry Sendik and Daniel Cohen-Or. Deep correlations for texture synthesis. ACM Transactions on Graphics (TOG), 36(5):161, 2017. 2

[39] Christian Szegedy, Sergey Ioffe, Vincent Vanhoucke, and Alexander A Alemi. Inception-v4, inception-resnet and the impact of residual connections on learning. In $A A A I$, volume 4, page 12, 2017. 7, 13

[40] Dmitry Ulyanov, Vadim Lebedev, Andrea Vedaldi, and Victor S Lempitsky. Texture networks: Feed-forward synthesis of textures and stylized images. In ICML, pages 1349-1357, 2016. 1, 2

[41] Li-Yi Wei and Marc Levoy. Fast texture synthesis using tree-structured vector quantization. In Proceedings of the 27th annual conference on Computer graphics and interactive techniques, pages 479-488. ACM Press/AddisonWesley Publishing Co., 2000. 2

[42] Yonatan Wexler, Eli Shechtman, and Michal Irani. Spacetime completion of video. IEEE Transactions on Pattern Analysis \& Machine Intelligence, (3):463-476, 2007. 2

[43] Chao Yang, Xin Lu, Zhe Lin, Eli Shechtman, Oliver Wang, and Hao Li. High-resolution image inpainting using multiscale neural patch synthesis. In The IEEE Conference on Computer Vision and Pattern Recognition (CVPR), volume 1, page 3, 2017. 2

[44] Jiahui Yu, Zhe Lin, Jimei Yang, Xiaohui Shen, Xin Lu, and Thomas S Huang. Free-form image inpainting with gated convolution. arXiv preprint arXiv:1806.03589, 2018. 2
[45] Jiahui Yu, Zhe Lin, Jimei Yang, Xiaohui Shen, Xin Lu, and Thomas S Huang. Generative image inpainting with contextual attention. arXiv preprint arXiv:1801.07892, 2018. 2, 7, 13

[46] Richard Zhang, Phillip Isola, Alexei A Efros, Eli Shechtman, and Oliver Wang. The unreasonable effectiveness of deep features as a perceptual metric. In $C V P R, 2018.7$

[47] Yang Zhou, Zhen Zhu, Xiang Bai, Dani Lischinski, Daniel Cohen-Or, and Hui Huang. Non-stationary texture synthesis by adversarial expansion. ACM Trans. Graph., 37(4):49:149:13, 2018. 2

[48] Jun-Yan Zhu, Taesung Park, Phillip Isola, and Alexei A Efros. Unpaired image-to-image translation using cycleconsistent adversarial networks. In Computer Vision (ICCV), 2017 IEEE International Conference on, 2017. 2

[49] Jun-Yan Zhu, Richard Zhang, Deepak Pathak, Trevor Darrell, Alexei A Efros, Oliver Wang, and Eli Shechtman. Toward multimodal image-to-image translation. In Advances in Neural Information Processing Systems, 2017. 2 


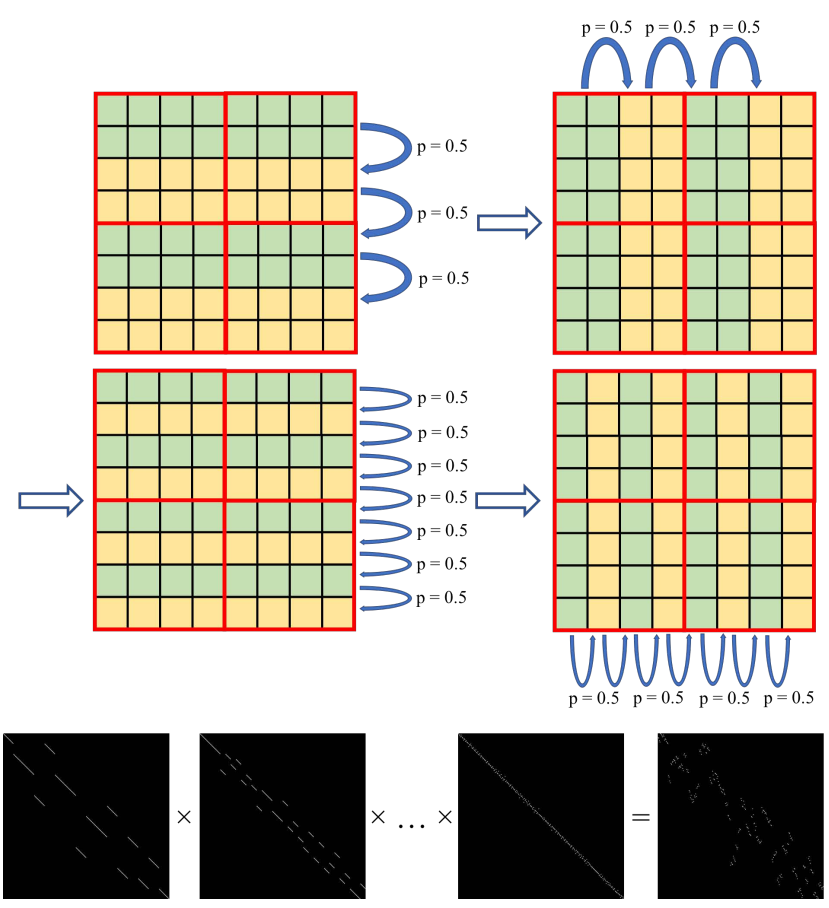

Figure 7. Our shuffling procedure. On the top figure we visualize the procedure example $P_{0} \circ P_{1}\left(T\left(z_{i}^{l}\right)\right)$, where $z_{i}^{l}$ is a $4 \times 4$ cell in a $2 \times 2$ grid framed in red. The procedure is composed of the random swapping operations between a green strip and its subsequently adjacent yellow strip in four directions: top-down, bottom-up, leftto-right, and right-to-left. The swapping operations start at scale 2 (the 1 st row) and then are repeated at scale 1 (the 2 nd row). The bottom figure (zoom-in to check) demonstrates the composition of swapping operations at several scales applied to an identity matrix. The resulting composed matrix can serve as a row permutation matrix left-multiplied to $T\left(z_{i}^{l}\right)$. Another similar matrix can serve as a column permutation matrix right-multiplied to $T\left(z_{i}^{l}\right)$. The row and column permutation matrices are independently sampled for each training iteration.

\section{Supplementary material}

\section{A. Shuffling procedure visualization}

We visualize our shuffling procedure in Figure 7.

\section{B. Texture palette and brush examples}

In order to diversify our applications, we, in addition, collected a plant texture dataset from Adobe Stock and randomly split it into 1,074 training and 119 testing images. We show the texture palette and brush application on the earth texture and plant texture datasets in Figure 8. Furthermore, we show in Figure 9 a camouflage effect of brush painting on the animal texture dataset, intentionally given the background patterns similar to brush patterns. It indicates the smooth interpolation over different textures. The dynamic processes of drawing such paintings plus the painting of Figure 1 in the main paper are demonstrated in
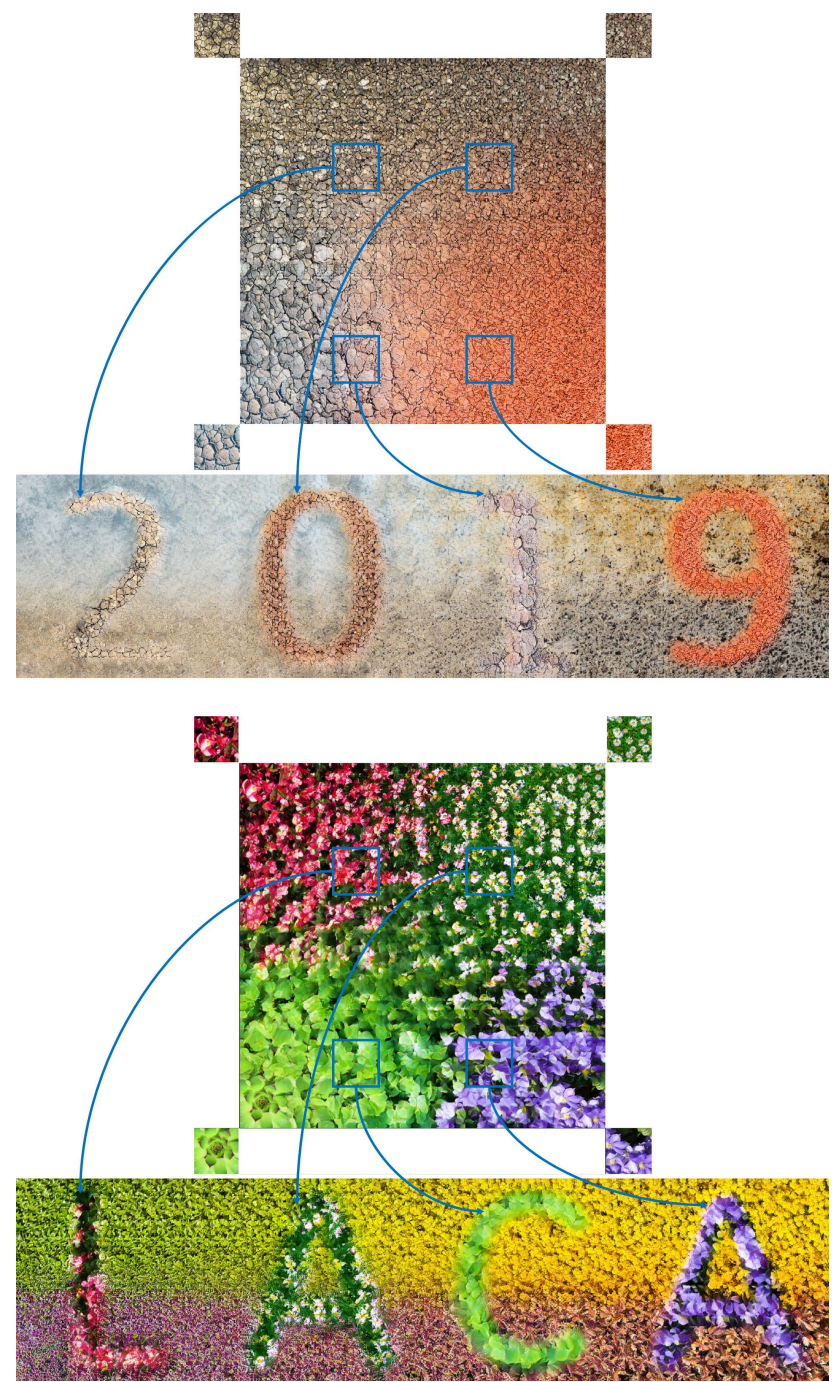

Figure 8 . Texture interpolation and texture painting using our network on the earth texture and plant texture datasets. The top part shows a $1024 \times 1024$ palette created by interpolating four source textures at the corners outside the palette. The bottom part shows a $512 \times 2048$ painting of letters with different textures sampled from the palette. The letters are interpolated by our method with the background, also generated by our interpolation.

the videos at GitHub. The videos are encoded using MP4 libx 265 codec at 60 frame rate and $16 \mathrm{M}$ bit rate.

\section{Texture dissolve examples}

We shows in Figure 10 additional sequences of video frame samples with gradually varying weights on the earth texture and plant texture datasets. The corresponding videos plus the video for Figure 3 in the main paper are at GitHub. The videos are encoded using MP4 libx265 codec at 60 frame rate and $16 \mathrm{M}$ bit rate. 


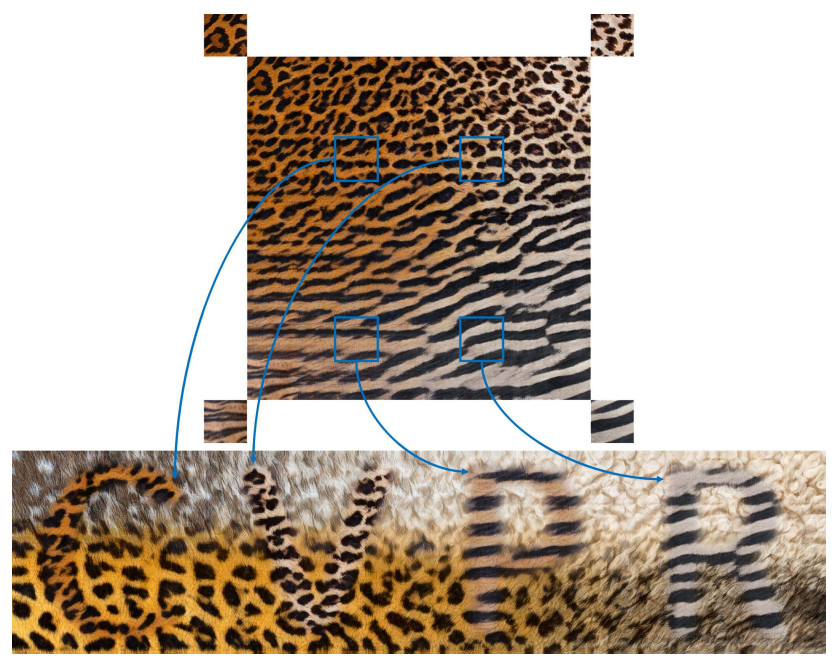

Figure 9. Texture interpolation and texture painting with camouflage effect using our network on the animal texture dataset. The top part shows a $1024 \times 1024$ palette created by interpolating four source textures at the corners outside the palette. The bottom part shows a $512 \times 2048$ painting of letters with different textures sampled from the palette. The letters are interpolated by our method with the background, also generated by our interpolation.

\section{Animal hybridization details and examples}

In Figure 11, we show and illustrate the pipeline to hybridize a dog and a bear by interpolating their furs in the hole for the transition region. Two additional results are shown in Figure 12.

\section{E. Network architecture details}

We set the texture image size to be 128 throughout our experiments. The proposed $E^{l}, E^{g}, D^{r e c}$, and $D^{i t p}$ architectures are employed or adapted from the discriminator architecture in [23], where layers with spatial resolutions higher than $128 \times 128$ are removed. We also adopt their techniques including pixel normalization instead of batch normalization, and leaky ReLU activation. The minibatch standard deviation channel is also preserved for $D^{r e c}$ and $D^{i t p}$, but not for $E^{l}$ and $E^{g}$. For $E^{l}$, we truncate the architecture so that the output local latent tensor is $m$ times smaller than the input texture, where $m=4$ in all our experiments. We tried using deeper architectures but noticed this does not favor reconstruction quality. For $E^{g}$, we truncate the architecture at $1 \times 1$ resolution right before the fullyconnected layer, because we are doing encoding rather than binary classification.

Our $G$ is modified from the fully-convolutional generator architecture from Karras et al. [23] with three changes. First, the architecture is truncated to accept an input spatial resolution that is $m=4$ times smaller than the texture size, and to output the original texture size. Second, the local and global latent tensor inputs are concatenated together along the channel dimension after they are fed into $G$. A third important point is that since our goal is to interpolate a larger texture image output, at the bottleneck layer the receptive field should be large enough to cover the size of input image. We do this by inserting a chain of five residual blocks [16] in the generator after local and global latent tensor concatenation and before the deconvolution layers from [23].

\section{F. Training details}

Our training procedure again follows the progressive growing training in [23], where $E^{l}, E^{g}, G, D^{r e c}$, and $D^{i t p}$ simultaneously grow from image spatial resolution at $32 \times 32$ to $128 \times 128$. We repeatedly alternate between performing one training iteration on $D^{r e c}$ and $D^{i t p}$, and then four training iterations on $E^{l}, E^{g}$, and $G$. At each intermediate resolution during growth, the stabilization stage takes 1 epoch of training and the transition stage takes 3 epochs. After the growth is completed, we keep training the model until a total of 20 epochs is reached.

We use Adam [25] as our optimization approach with no exponential decay rate $\beta_{1}=0.0$ for the first moment estimates and with the exponential decay rate for the second moment estimates $\beta_{2}=0.99$. The learning rate is set to 0.001 before the model grows to the final resolution $128 \times$ 128 and then is set to 0.0015 at $128 \times 128$. The trainable weights of the autoencoder and discriminator are initialized with the equalized learning rate technique from [23]. We train and test all our models on 8 NVIDIA GeForce GTX $1080 \mathrm{Ti}$ GPUs with $12 \mathrm{~GB}$ of GPU memory each. Based on the memory available and the training performance, we set the batch size at 64 , and the training lasts for 3 days.

The weights of losses is not sensitive to the dataset. We simply set them to balance the order of magnitude of each loss: $\lambda_{1}=100, \lambda_{2}=\lambda_{4}=0.001$, and $\lambda_{3}=\lambda_{5}=1$.

\section{G. Experimental evaluation details}

Seam classifier. The architecture and training details of seam classifier are almost the same as those of $D^{r e c}$ and $D^{i t p}$ except (1) we remove the minibatch standard deviation channel, (2) we add a sigmoid activation layer after the output layer for the binary cross-entropy loss computation, and (3) we exclude the progressive growing process. We directly use the sigmoid output of the classifier as the seam score for each input image.

Repetition classifier. The architecture and training details of repetition classifier are almost the same as those of the seam classifier except the input image size is $128 \times 256$ instead of $128 \times 128$, where the negative examples are random crops of size $128 \times 256$ from real datasets and the positive examples are horizontally tiled twice from random crops of size $128 \times 128$ from real datasets.

Inception model finetuning. Our inception scores are computed from the state-of-the-art Inception-ResNet- $v 2$ in- 

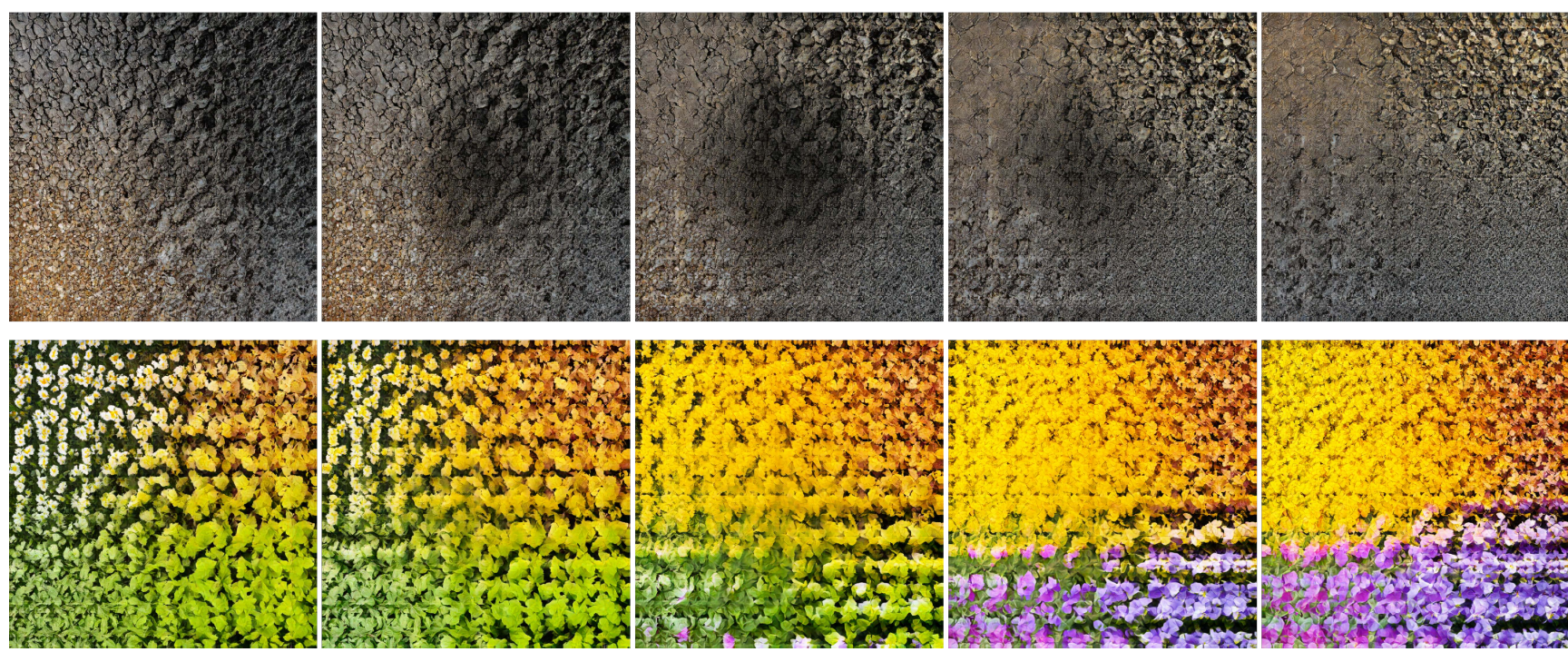

Figure 10. Sequences of dissolve video frame samples with size $1024 \times 1024$ on the earth texture and plant texture datasets, where each frame is also with effect of interpolation.

ception model architecture [39] finetuned with our two datasets separately.

\section{H. Baseline method details}

Naïve $\alpha$-blending. We split the output into 8 square tiles, where the end textures are copied as-is, and the intervening tiles (copies of the two boundaries) are linearly per-pixel $\alpha$-blended.

Image Melding [8]. We selected Image Melding in its inpainting mode as a representative of patch-based methods. We use the default setting of the official public implementation $^{2}$.

AdaIN [19]. Style transfer techniques can potentially be leveraged for the interpolation task by using random noise as the content image and texture sample as the style. We interpolate the neural features of the two source textures to vary the style from left to right. We consider AdaIN as one representative of this family of techniques, as it can run with arbitrary content and style images. However, with the default setting of the official implementation ${ }^{3}$ and their pre-trained model, AdaIN has some systematic artifacts as it over-preserves the noise appearance. Therefore, we only show qualitative results in Figure 4 in the main paper, and in Figure 15 to Figure 19 here. We did not include this method in the quantitative evaluation.

WCT [31]. WCT is an advancement over AdaIN with whitening and coloring transforms (WCT) as the stylization technique and works better on our data. We use its official public implementation ${ }^{4}$ with default setting and their pre-

\footnotetext{
${ }^{2}$ https://www.ece.ucsb.edu/ psen/melding

3 https://github.com/xunhuang1995/AdaIN-style

${ }^{4}$ https://github.com/Yijunmaverick/ UniversalstyleTransfer
}

trained model. By design, this method does not guarantee accurate reconstruction of input samples.

DeepFill [45]. Texture interpolation can be considered an instance of image hole-filling. The training code for the most recent work in this area [32] is not released yet. We, therefore, tried another recent method called DeepFill [45] with their official code ${ }^{5}$. We re-trained it for our two texture datasets separately with $256 \times 256$ input image size, $128 \times 128$ hole size, and all the other default settings. The interpolation results suffered from two major problems: (i) the method is not designed for inpainting wide holes (in our experiment $128 \times 768$ ) because of lack of such wide ground truth; (ii) even for a smaller hole with size $128 \times 128$, as shown in the failure cases in Figure 4 in the main paper and in Figure 15 to Figure 19, this work systematically failed to merge the two source textures gradually. We, therefore, excluded this method from our quantitative comparisons.

PSGAN [3]. The most closely related work to ours, PSGAN, learns a smooth and complete neural manifold that favors interpolation. However, it only supports constraining the interpolation in latent space, and lacks a mechanism to specify end texture conditions using image examples. To allow for a comparison, we have trained a PSGAN model for each of our datasets separately, using the official code ${ }^{6}$ and default settings. Then, we optimize for the latent code that corresponds to each of the end texture images by backpropagating through L-BFGS-B [5]. We use the gradients of the $L_{1}$ reconstruction loss and the Gram matrix loss [22] and initialize randomly the latent vectors. We use 100 different initializations and report the best result.

\footnotetext{
${ }^{5}$ https://github.com/JiahuiYu/generative_ inpainting

${ }^{6}$ https://github.com/zalandoresearch/psgan
} 


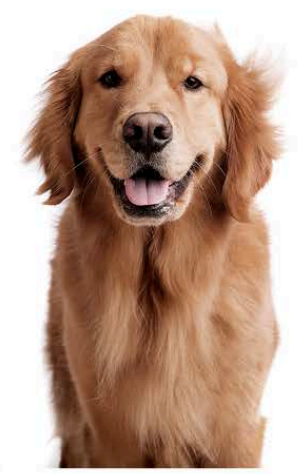

(a) The original dog

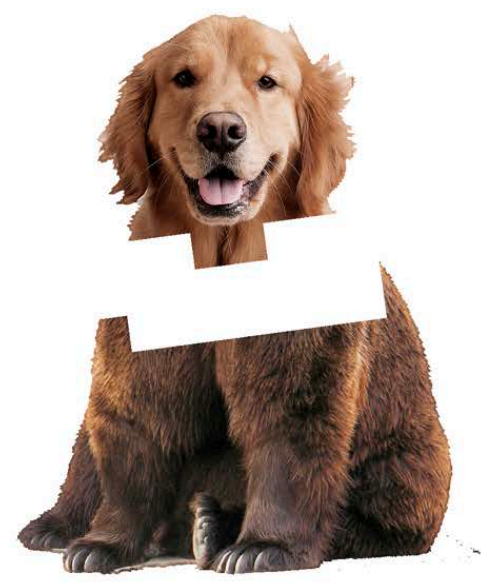

(d) Rasterized hole

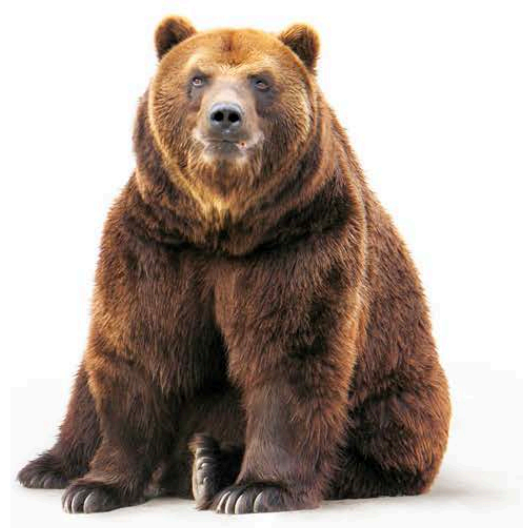

(b) The original bear

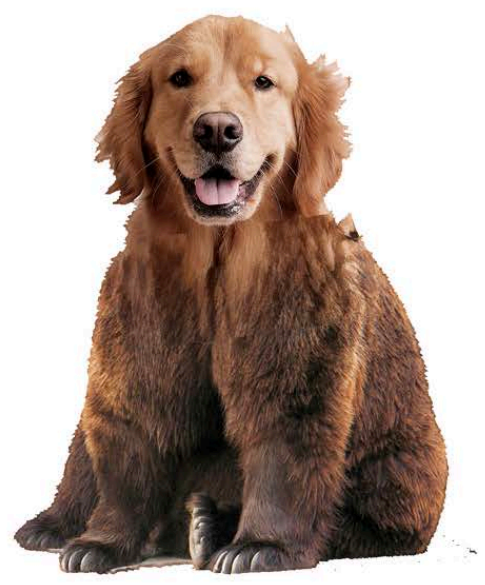

(e) Interpolation

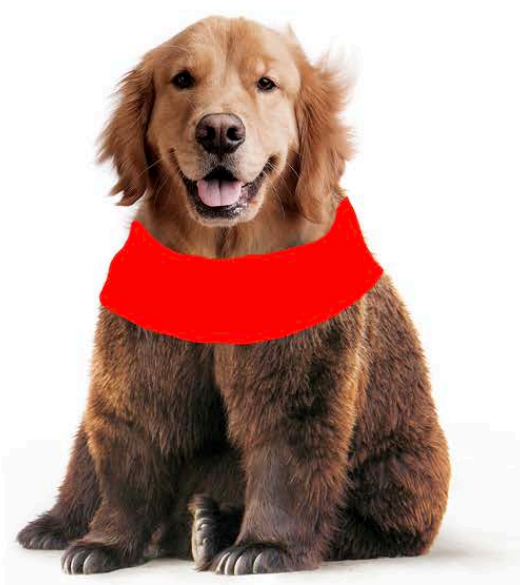

(c) Input: aligned animal regions with hole

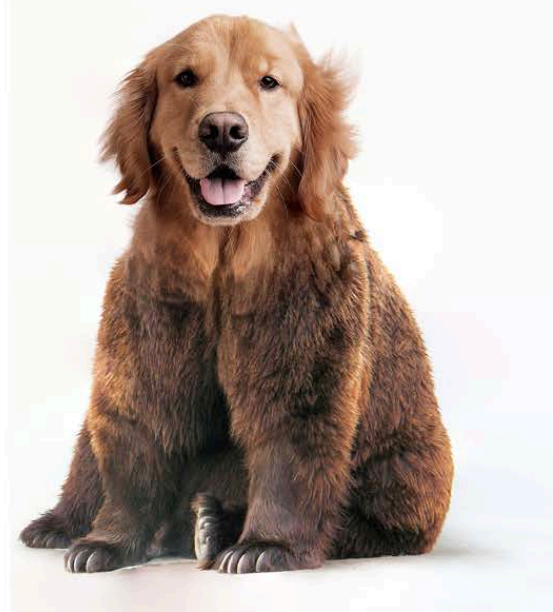

(f) Graph cuts and Poisson blending postprocessing

Figure 11. Animal hybridization pipeline. (a) and (b) are two original images. (c) is the input to the pipeline, composed of the aligned regions of (a) and (b) in the same image and the hole for the transition region. (d) shows that we rasterize the hole because Texture Mixer works on square patches. The patch size is $128 \times 128$. (e) shows that we interpolate in the rasterized hole region using adjacent texture patches, and then composite this back on top of the original image. This involves two details: (1) if a texture patch covers background, those background pixels are replaced by foreground pixels using the Content-Aware Fill function in Photoshop; and (2) we blend latent tensors between two images using spatially varying weights. (f) We use graph cuts [27] and standard Poisson blending [34] to postprocess the boundaries.

\section{More qualitative comparisons}

More qualitative comparisons are shown from Figure 15 to Figure 19. They are all used for quantitative comparison and user study as reported in Table 1 in the main paper. In addition, Figure 20 demonstrates one of our failure examples when dealing with strong structural textures.

\section{J. User study details}

Our user study webpage is shown in Figure 13. To guarantee the accuracy of users' feedback, we insert sanity check by comparing our interpolation results with another naive baseline results where the transition regions are filled with constant pixel values. The constant value is computed as the mean value of the two end texture pixels, as shown in Figure 14. The preference should be obvious and deterministic without subjective variance. In our statistics, only two users made a mistake once on the sanity check questions. We then manually checked their answers to other real questions but didn't notice any robot or laziness style. We there trust and accept all users' feedback. 


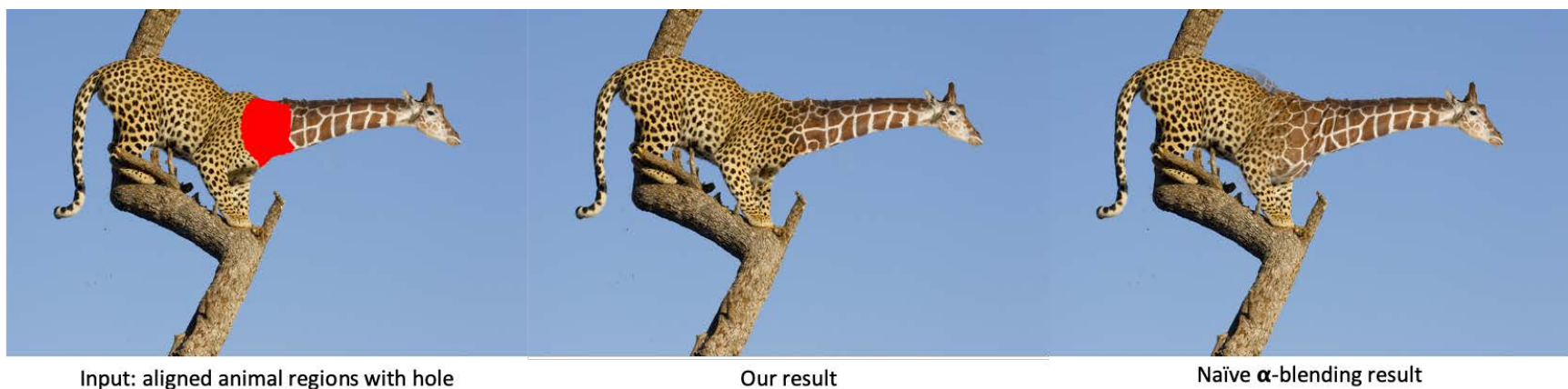

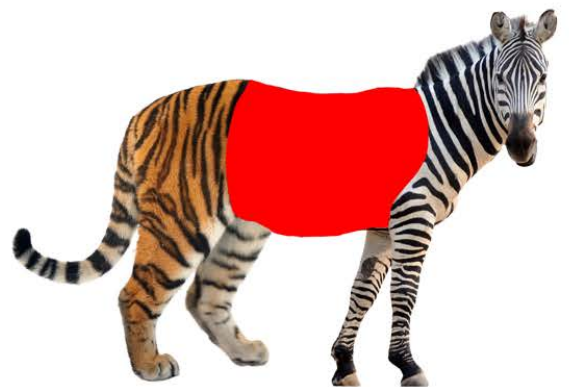

Input: aligned animal regions with hole
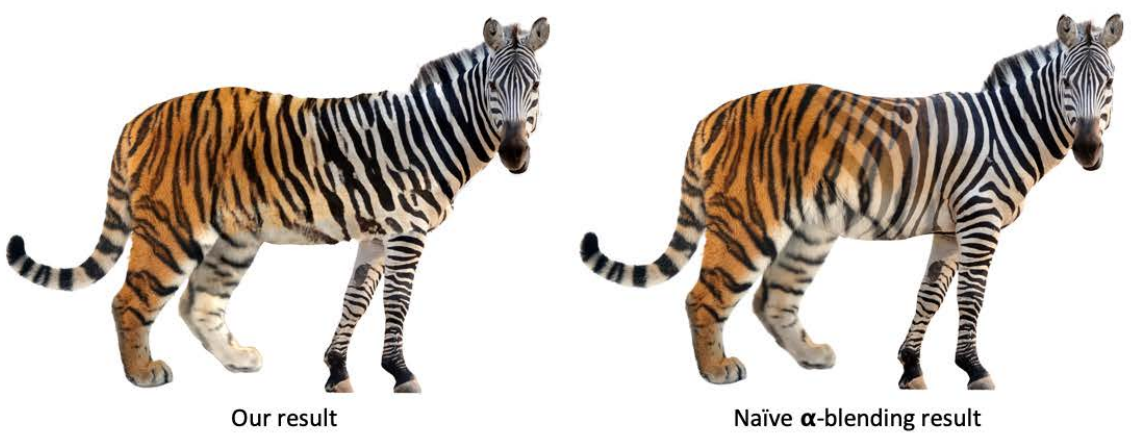

Naïve $\boldsymbol{\alpha}$-blending result

Figure 12. Two animal hybridization examples. The top image is in the size $2636 \times 3954$ and the bottom image is in the size $2315 \times 2664$ Our interpolations between the two animal furs is smoother, has less ghosting, and is more realistic than those of the Naïve $\alpha$-blending.

\section{Texture Image Transition Study}

For this task, we would like to know which image shows better appearance of texture transition from left to right.

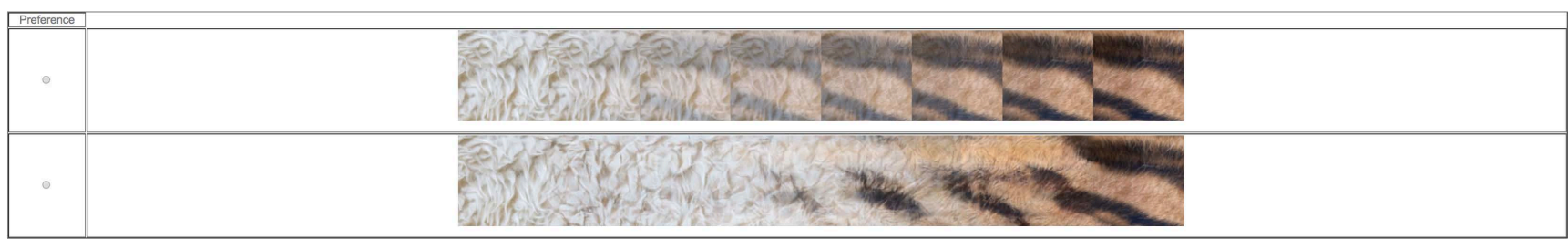

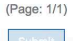

Figure 13. User study webpage design. 


\section{Texture Image Transition Study}

For this task, we would like to know which image shows better appearance of texture transition from left to right.

When comparing images please pay attention to:

Undesired repetitions;

Undesired cuts in the texture

Undesired blurriness;

Transitions between the two textures should be ideally smooth \& gradual.

We may reject submissions with suspiciously short working time and workers who misjudge obvious comparisons we have intentionally included

A safe working time is $>7$ seconds per submission according to statistics.

Time limit per HIT: 2 minutes.

We suggest you choose the "Auto-accept next HIT" button on the top of the page.

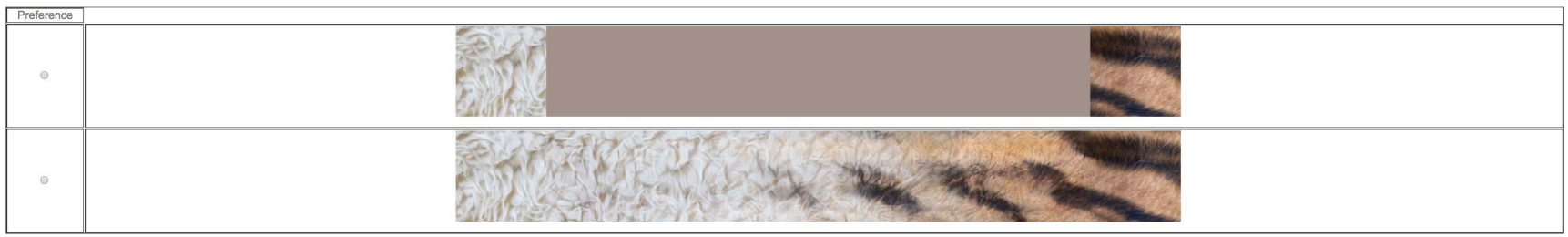

\begin{aligned} (Page: 1/1) \\ \hline \\ \hline\end{aligned}

Figure 14. A user study with sanity check where the preference should be obvious and deterministic without subjective variance. 


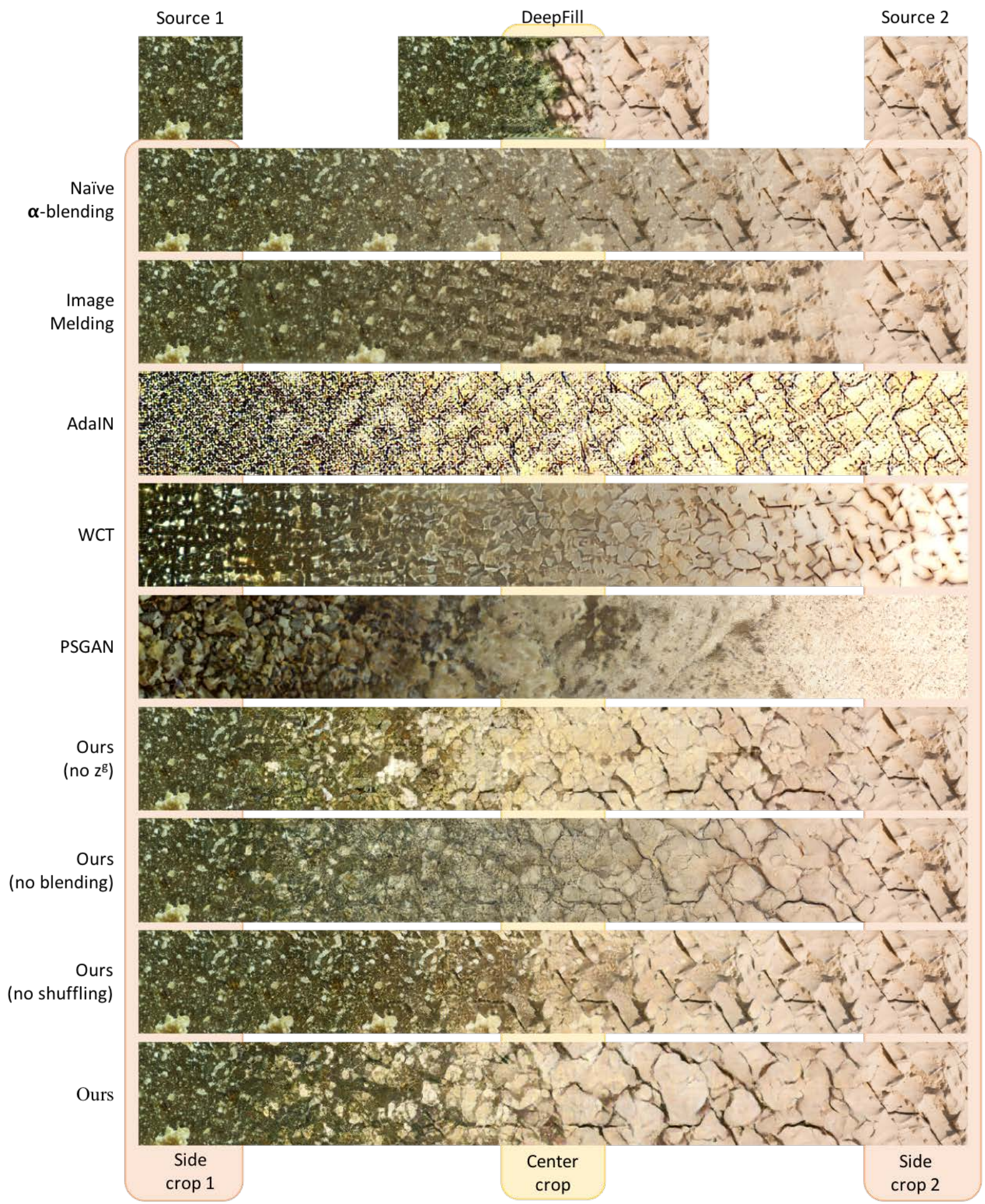

Figure 15. Qualitative demonstrations and comparisons of horizontal interpolation in the size of $128 \times 1024$ on the earth texture samples. We use the two side crops with the orange background for SPD measurement, and the center crop with the light yellow background for the other proposed quantitative evaluations. 


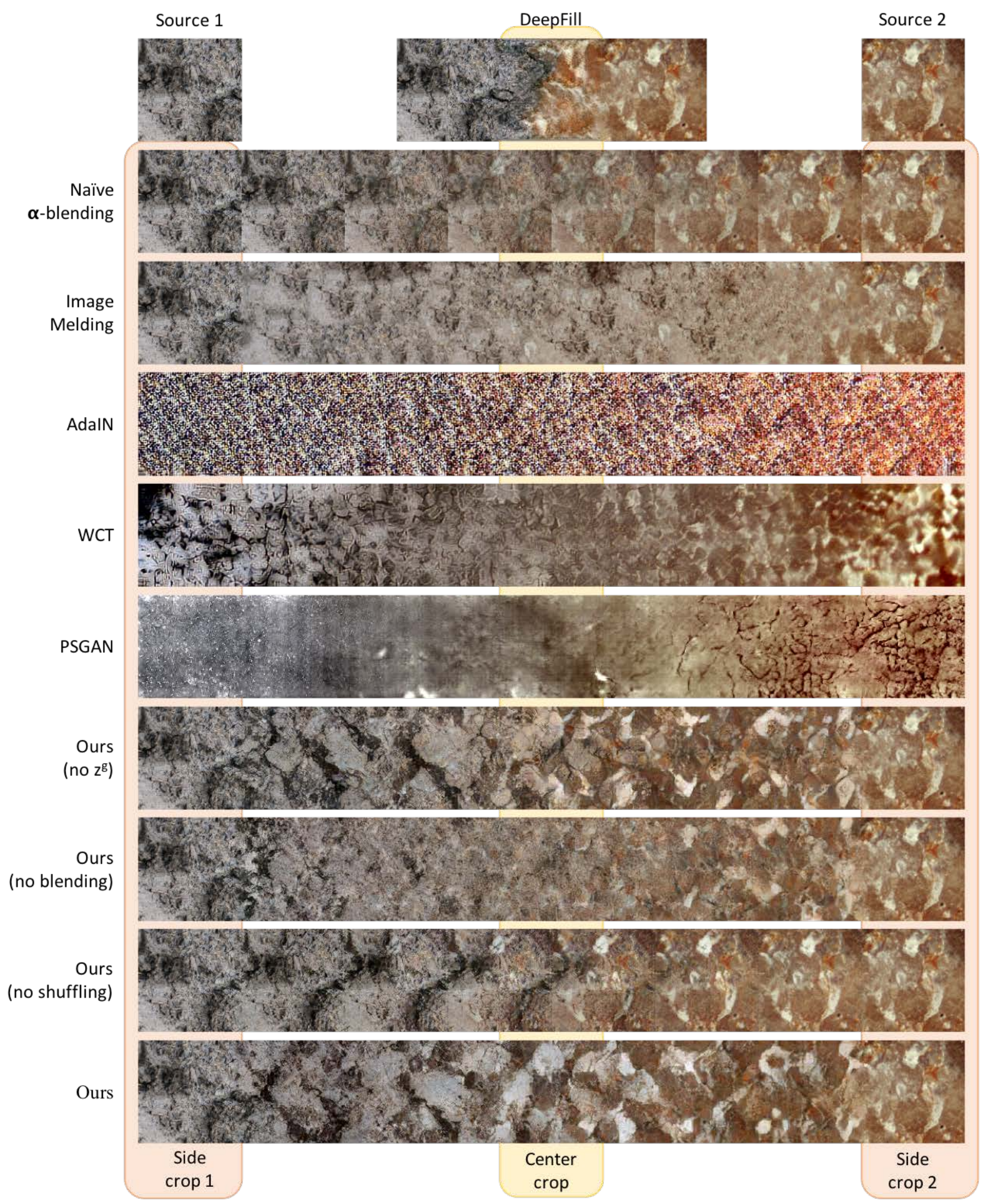

Figure 16. Qualitative demonstrations and comparisons of horizontal interpolation in the size of $128 \times 1024$ on the earth texture samples. We use the two side crops with the orange background for SPD measurement, and the center crop with the light yellow background for the other proposed quantitative evaluations. 


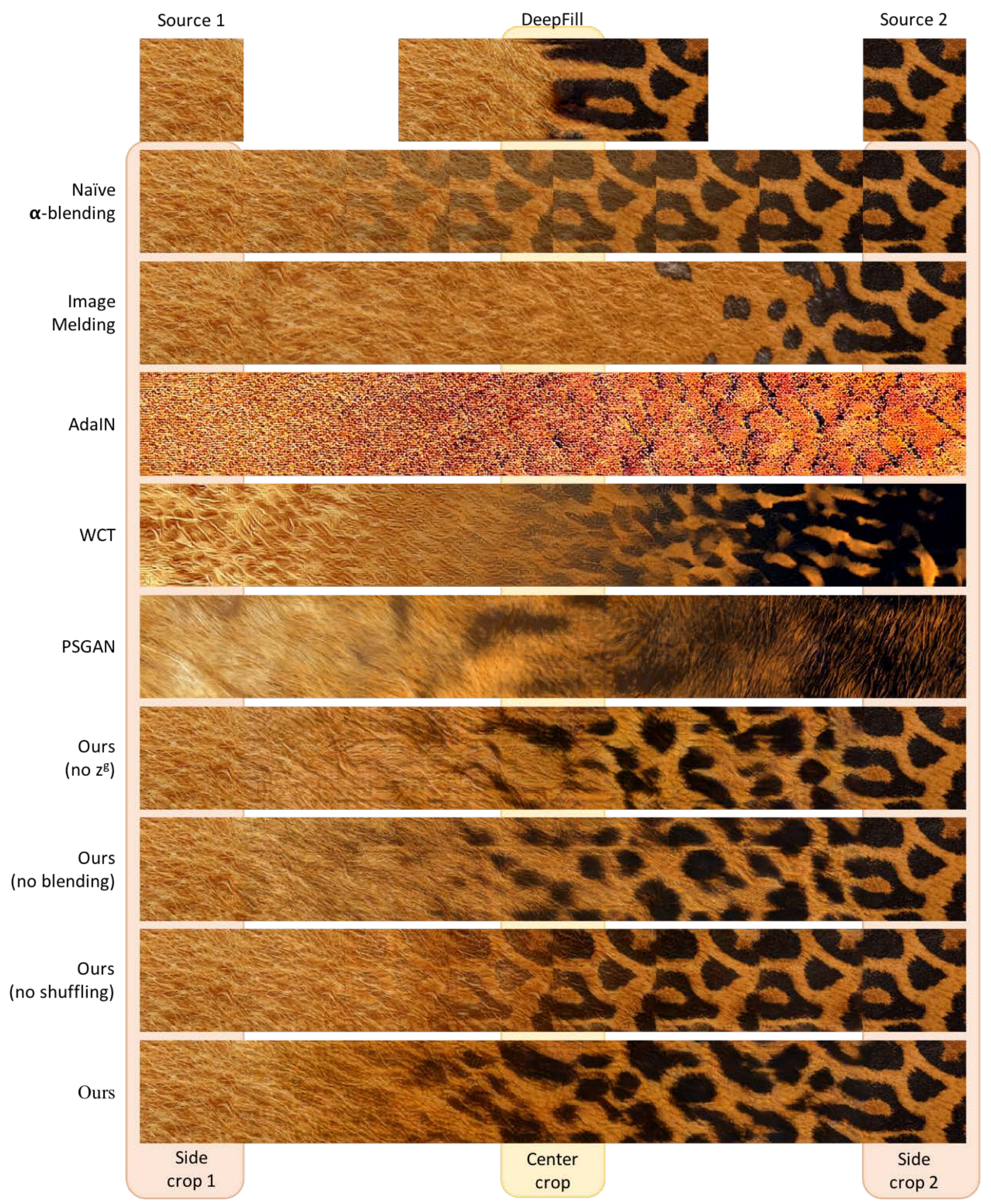

Figure 17. Qualitative demonstrations and comparisons of horizontal interpolation in the size of $128 \times 1024$ on the animal texture samples. We use the two side crops with the orange background for SPD measurement, and the center crop with the light yellow background for the other proposed quantitative evaluations. 


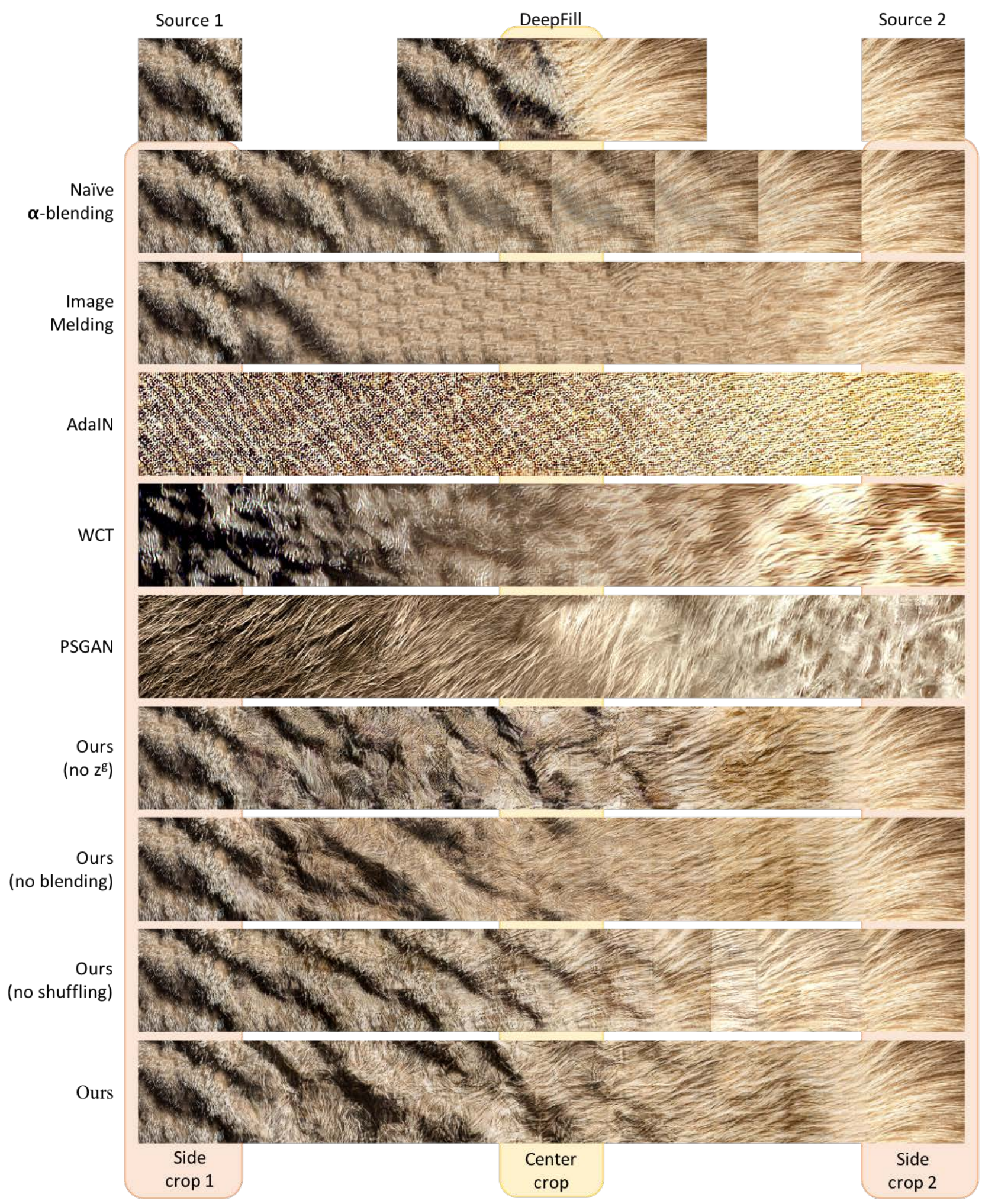

Figure 18. Qualitative demonstrations and comparisons of horizontal interpolation in the size of $128 \times 1024$ on the animal texture samples. We use the two side crops with the orange background for SPD measurement, and the center crop with the light yellow background for the other proposed quantitative evaluations. 


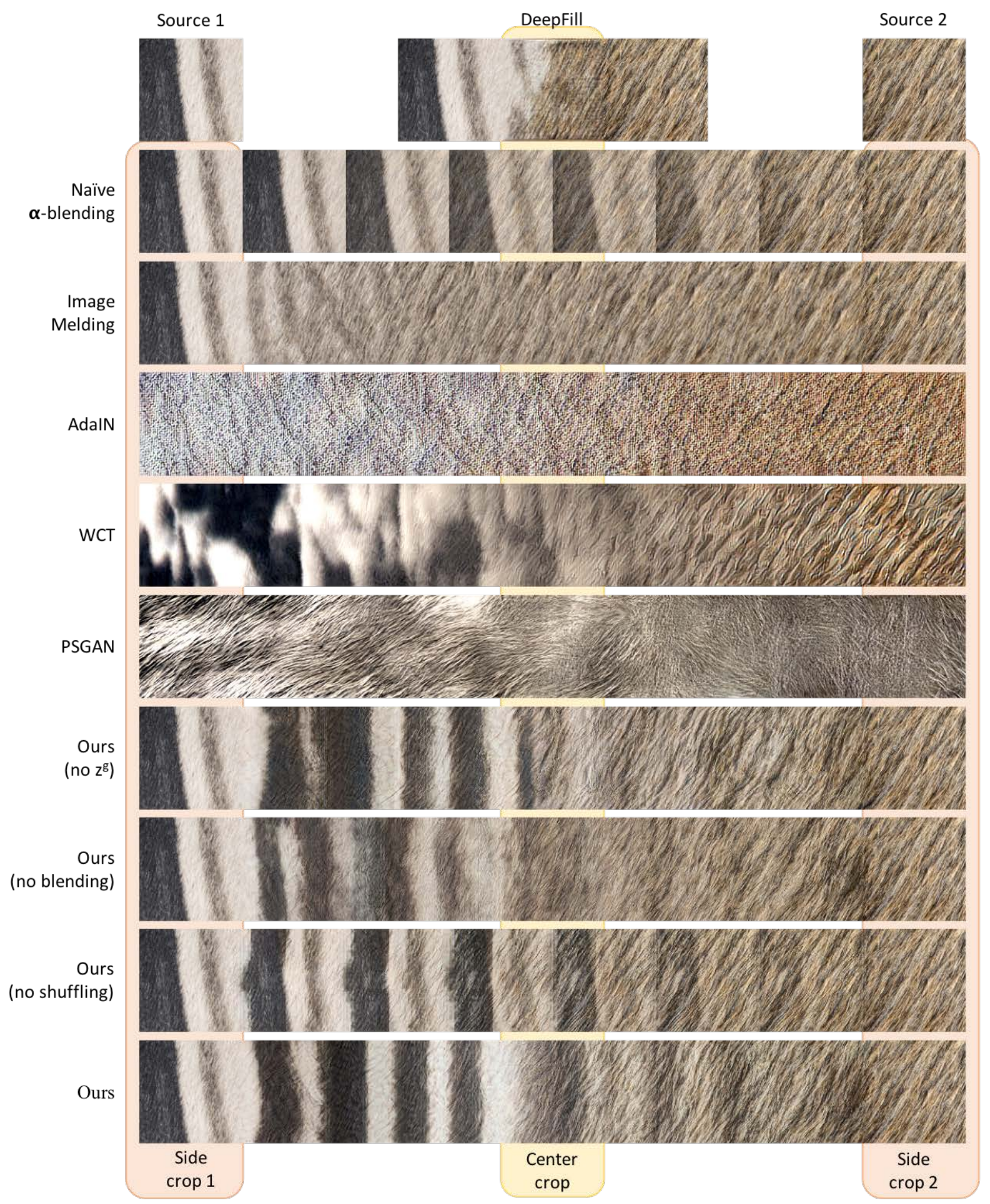

Figure 19. Qualitative demonstrations and comparisons of horizontal interpolation in the size of $128 \times 1024$ on the animal texture samples. We use the two side crops with the orange background for SPD measurement, and the center crop with the light yellow background for the other proposed quantitative evaluations. 


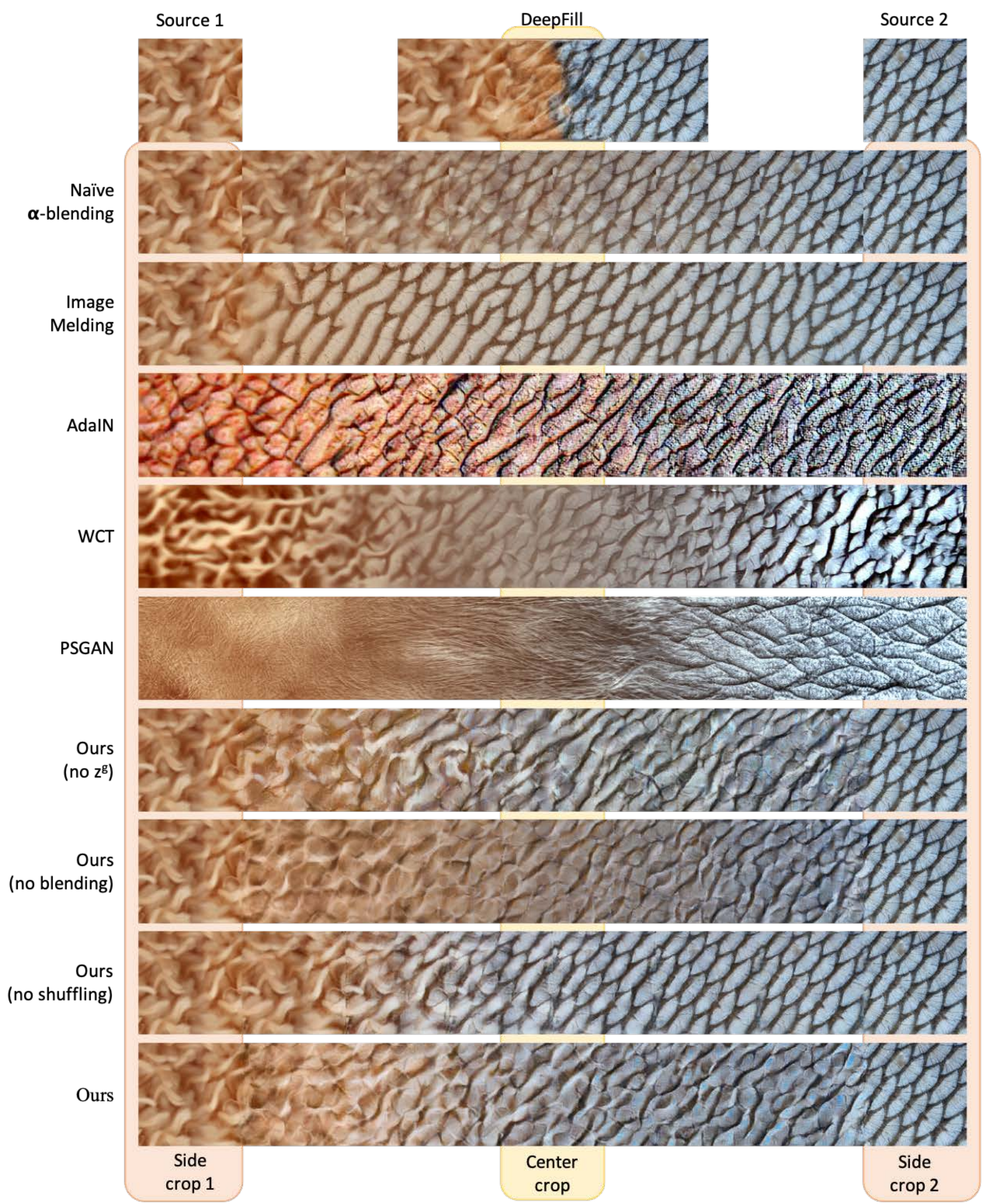

Figure 20. A qualitative demonstration of one of our failure examples. When dealing with strong structural source texture on the right, our full method didn't outperform ours without random shuffling during training, and didn't outperform Image Melding either. 